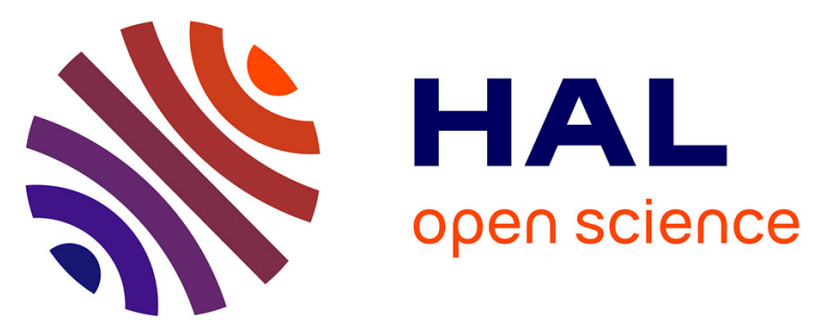

\title{
Genome-wide bioinformatic analyses predict key host and viral factors in SARS-CoV-2 pathogenesis
}

Mariana G Ferrarini, Avantika Lal, Rita Rebollo, Andreas J Gruber, Andrea Guarracino, Itziar Martinez Gonzalez, Taylor Floyd, Daniel Siqueira de Oliveira, Justin Shanklin, Ethan Beausoleil, et al.

\section{To cite this version:}

Mariana G Ferrarini, Avantika Lal, Rita Rebollo, Andreas J Gruber, Andrea Guarracino, et al.. Genome-wide bioinformatic analyses predict key host and viral factors in SARS-CoV-2 pathogenesis. Communications Biology, 2021, 4 (1), 10.1038/s42003-021-02095-0 . hal-03237937

\section{HAL Id: hal-03237937 https://hal.science/hal-03237937}

Submitted on 27 May 2021

HAL is a multi-disciplinary open access archive for the deposit and dissemination of scientific research documents, whether they are published or not. The documents may come from teaching and research institutions in France or abroad, or from public or private research centers.
L'archive ouverte pluridisciplinaire HAL, est destinée au dépôt et à la diffusion de documents scientifiques de niveau recherche, publiés ou non, émanant des établissements d'enseignement et de recherche français ou étrangers, des laboratoires publics ou privés.

\section{(c)(1)}

Distributed under a Creative Commons Attribution| 4.0 International License 


\title{
communications biology
}

ARTICLE

https://doi.org/10.1038/s42003-021-02095-0

\section{Genome-wide bioinformatic analyses predict key host and viral factors in SARS-CoV-2 pathogenesis}

\author{
Mariana G. Ferrarini (1) 1,11, Avantika Lal (1) 2,11, Rita Rebollo1, Andreas J. Gruber ${ }^{3}$, Andrea Guarracino (1) 4, \\ Itziar Martinez Gonzalez ${ }^{5}$, Taylor Floyd ${ }^{6}$, Daniel Siqueira de Oliveira7 ${ }^{7}$ Justin Shanklin ${ }^{8}$, Ethan Beausoleil ${ }^{8}$, \\ Taneli Pusa9 ${ }^{9}$, Brett E. Pickett (1D ${ }^{8 \times} \&$ Vanessa Aguiar-Pulido (10) ${ }^{10 凶}$
}

The novel betacoronavirus severe acute respiratory syndrome coronavirus 2 (SARS-CoV-2) caused a worldwide pandemic (COVID-19) after emerging in Wuhan, China. Here we analyzed public host and viral RNA sequencing data to better understand how SARS-CoV-2 interacts with human respiratory cells. We identified genes, isoforms and transposable element families that are specifically altered in SARS-CoV-2-infected respiratory cells. Wellknown immunoregulatory genes including CSF2, IL32, IL-6 and SERPINA3 were differentially expressed, while immunoregulatory transposable element families were upregulated. We predicted conserved interactions between the SARS-CoV-2 genome and human RNA-binding proteins such as the heterogeneous nuclear ribonucleoprotein A1 (hnRNPA1) and eukaryotic initiation factor 4 (elF4b). We also identified a viral sequence variant with a statistically significant skew associated with age of infection, that may contribute to intracellular host-pathogen interactions. These findings can help identify host mechanisms that can be targeted by prophylactics and/or therapeutics to reduce the severity of COVID-19.

\footnotetext{
${ }^{1}$ University of Lyon, INSA-Lyon, INRA BF2I, Villeurbanne, France. ${ }^{2}$ NVIDIA Corporation, Santa Clara, CA, USA. ${ }^{3}$ Department of Biology, University of Konstanz, Konstanz, Germany. ${ }^{4}$ Centre for Molecular Bioinformatics, Department of Biology, University Of Rome Tor Vergata, Rome, Italy. ${ }^{5}$ Amsterdam UMC, Amsterdam, The Netherlands. ${ }^{6}$ Center for Neurogenetics, Weill Cornell Medicine, Cornell University, New York, NY, USA. ${ }^{7}$ Laboratoire de Biométrie et Biologie Evolutive, Université de Lyon, Université Lyon 1, CNRS UMR 5558, Villeurbanne, France. ${ }^{8}$ Brigham Young University, Provo, UT, USA.

${ }^{9}$ Luxembourg Centre for Systems Biomedicine, Belvaux, Luxembourg. ${ }^{10}$ Department of Computer Science, University of Miami, Coral Gables, FL, USA.




n December of 2019, a novel betacoronavirus that was named severe acute respiratory syndrome coronavirus 2 (SARS-CoV-2) emerged in Wuhan, China ${ }^{1,2}$. This virus is responsible for causing the coronavirus disease of 2019 (COVID-19) and by 24 January 2021, it had already infected more than 95 million people worldwide, accounting for at least 2 million deaths ${ }^{3}$. The SARSCoV-2 genome is phylogenetically distinct from the SARS-CoV and Middle East Respiratory Syndrome (MERS-CoV) betacoronaviruses that caused human outbreaks in 2002 and 2012, respectively ${ }^{4,5}$. Based on its high sequence similarity to a coronavirus isolated from bats ${ }^{6}$, SARS-CoV-2 is hypothesized to have originated from bat coronaviruses, potentially using pangolins as an intermediate host before infecting humans ${ }^{7}$.

It remains a global priority to develop effective treatments for COVID-19, including treatments that inhibit viral replication inside human cells. At the same time, it is critical to control the hyper-inflammatory state that is frequently caused by this infection ${ }^{8}$. It is therefore important to define the biological processes that occur early in infection, including the mechanisms of viral replication, transcription, and translation inside host cells, which can be targeted by therapeutics ${ }^{9}$, as well as host immune responses that can be modulated ${ }^{8}$. Although many aspects of SARS-CoV-2 infection may be shared with other respiratory viruses, it is particularly interesting to identify its specific molecular interactions with host cells, to explain the unique clinical and epidemiological features of COVID-1910,11. Further, the observation of heterogeneous immune responses in COVID-19 patients $^{12}$ emphasizes the importance of identifying molecular responses to SARS-CoV-2, which are consistent across patients, and can therefore be targeted to develop widely applicable treatments.

SARS-CoV-2 enters human cells by binding to the angiotensinconverting enzyme 2 (ACE2) receptor ${ }^{13}$. Once inside the infected cell, components of the virus interact with host cell machinery. Coronaviruses have been shown to co-opt a diverse range of host factors for their life cycle, forming both protein-protein interactions and RNA-protein interactions with host factors ${ }^{14,15}$. Furthermore, viruses generally trigger a drastic host response during infection. A subset of these specific changes in gene regulation are associated with viral replication and, therefore, can be seen as potential drug targets. In addition, transposable element (TE) overexpression has been observed upon viral infection ${ }^{16}$ and TEs have been actively implicated in gene regulatory networks related to immunity ${ }^{17}$.

Recent studies have sought to understand the molecular interactions between SARS-CoV-2 and infected cells ${ }^{18,19}$, and some have quantified gene expression changes in patient samples or cultured lung-derived cells infected by SARS-CoV-2 $20-22$. However, it remains important to contrast the effects of SARSCoV-2 with those of other respiratory viruses. Furthermore, host factors such as TEs and genetic isoforms remain understudied in the context of SARS-CoV-2 infection. Here we aim to identify host factors, pathways, and processes that are altered in response to SARS-CoV-2 infection in human cells, in particular those that are unaffected by other respiratory infections. Moreover, although many previous studies have examined immune cells, we focused specifically on human airway epithelial cells, as they are the primary entry points for respiratory viruses and therefore constitute the first producers of inflammatory signals that, in addition to their antiviral activity, promote the initiation of the innate and adaptive immune responses.

We identified a signature of altered gene expression that is consistent across published datasets of SARS-CoV-2-infected human lung cells. We present extensive results from functional analyses (signaling pathway enrichment, biological functions, transcript isoform usage, and TE overexpression) of the genes differentially expressed during SARS-CoV-2 infection ${ }^{22}$, highlighting a consistent isoform switch of the $I L-6$ gene in SARSCoV-2-infected cell lines. We also analyzed viral genome sequences to predict specific interactions between the SARS-CoV2 RNA genome and human proteins that may be involved in viral replication, transcription, or translation, and identified at least one viral sequence variation that is significantly associated with patient age in humans. Knowledge of these molecular and genetic mechanisms is important to understand SARS-CoV-2 pathogenesis and to improve the future development of effective prophylactic and therapeutic treatments.

\section{Results}

We designed a comprehensive bioinformatics workflow to identify relevant host-pathogen interactions using a complementary set of computational analyses (Fig. 1). First, we carried out an exhaustive analysis of differential gene expression in human lung cells infected by SARS-CoV-2 or other respiratory viruses, identifying gene-, isoform-, and pathway-level responses, which specifically characterize SARS-CoV-2 infection. Second, we predicted putative interactions between the SARS-CoV-2 RNA genome and human RNA-binding proteins (RBPs). Third, we identified a subset of these human RBPs, which are also differentially expressed in response to SARS-CoV-2. Finally, we identified a viral sequence variant that could play a role in intracellular host-pathogen interaction.

SARS-CoV-2 infection elicits a specific gene expression and pathway signature in human cells. We wanted to identify genes that were differentially expressed across multiple SARS-CoV-2infected samples, while not significant in samples infected with other respiratory viruses. As a primary dataset, we selected GSE14750722 (Fig. 2a), which includes gene expression measurements from three cell lines derived from the human respiratory system (NHBE, A549, Calu-3) infected either with SARS-CoV-2, influenza A virus (IAV), respiratory syncytial virus (RSV), or human parainfluenza virus 3 (HPIV3). We also analyzed an additional dataset GSE150316 (Fig. 2a), which includes RNA sequencing (RNA-seq) extracted from formalin-fixed, paraffin-embedded (FFPE) histological sections of lung biopsies from COVID-19 deceased patients and healthy individuals. This second dataset encompasses a variable number (1-5) of postmortem lung biopsies per subject. The results coming from FFPE sections were less consistent, presumably due to the collection of biospecimens from different sites within the lung. Supplementary Data 1 provides details of all the samples included in our analyses.

We retrieved 41 differentially expressed genes (DEGs) that showed significant and consistent expression changes in at least three datasets from cell lines infected with SARS-CoV-2 and were not significantly affected in cell lines infected with other viruses within the same dataset. To these, we added 36 genes that showed significant and consistent expression changes in 2 of 4 cell line datasets infected with SARS-CoV-2 and at least 1 lung biopsy sample from a SARS-CoV-2 patient. The rationale behind these criteria was that results from FFPE sections were less reliable and, hence, were used only as supporting evidence where a gene was altered in at least two cell line samples. We further excluded four discordant genes that were upregulated in more than one cell line sample and downregulated in the biopsy samples. Thus, the final set consisted of 73 DEGs (Supplementary Data 2a): 53 upregulated and 20 downregulated, of which 41 had an absolute $\log 2 \mathrm{FC}>1$ in at least one dataset (selected genes from this list are shown in Table 1).

SERPINA3, an antichymotrypsin that was proposed as a candidate inhibitor of viral replication ${ }^{23}$, was the only gene 


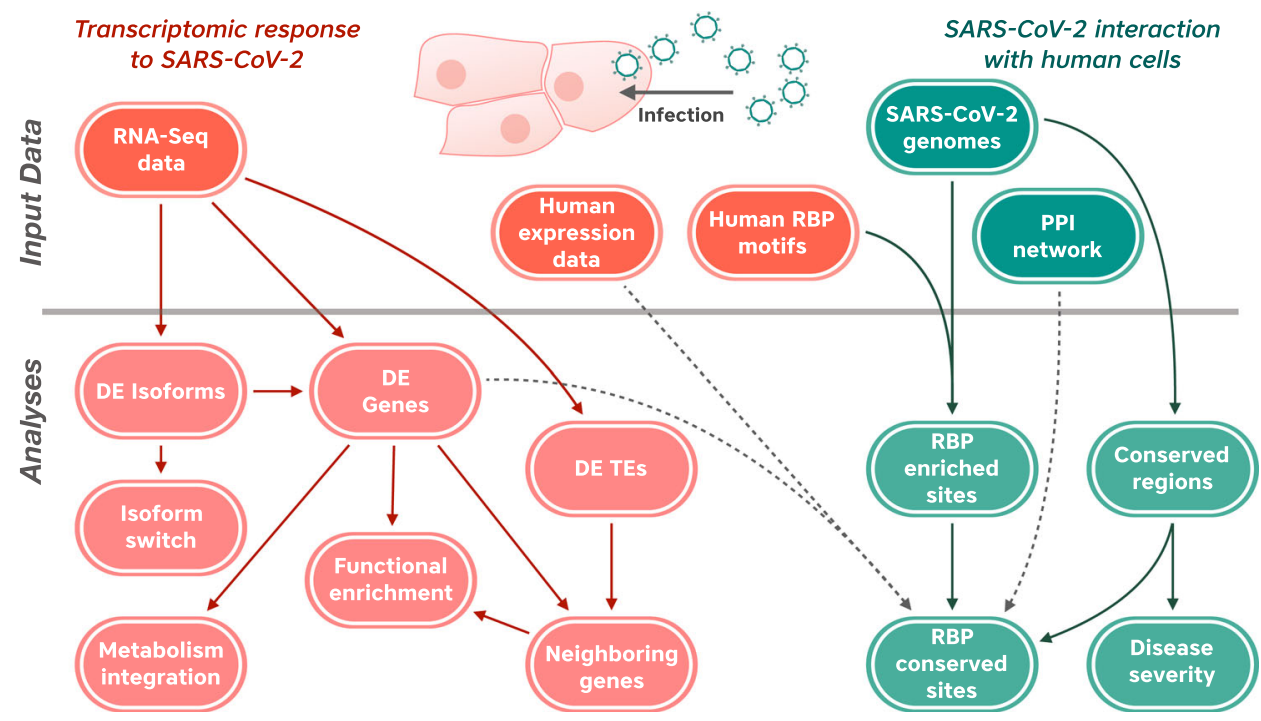

Fig. 1 Overview of the bioinformatic workflow applied in this study. As indicated in orange, RNA-seq data from SARS-CoV-2-infected samples were used as the input to identify differentially expressed (DE) genes, isoforms, and transposable elements (TEs). DE genes were used to identify functional enrichment of deregulated genes and possible impacts on metabolism. Neighboring genes of differentially expressed TEs (DETEs) were analyzed to verify if TEs could serve as regulatory mechanisms of gene expression. In green, the complete genome of the SARS-CoV-2 virus was used to identify enrichment of RNA-binding protein (RBP) motifs. We also used all available sequenced genomes as of 11 November 2020, to detect conserved RBP motifs and possible links to disease severity.

specifically upregulated in the four cell line datasets tested (Table 1). Other interesting upregulated genes were the amidohydrolase $V N N 2$, the pro-fibrotic gene $P D G F B$, the $\beta$ interferon (IFN) regulator PRDM1 and the proinflammatory cytokines CSF2 and IL32. FKBP5, a known regulator of nuclear factor- $\mathrm{kB}$ (NF- $\mathrm{kB}$ ) activity, was among the consistently downregulated genes. This set of genes represents a signature of host response specific to SARS-CoV-2 and may help to explain the specific clinical and epidemiological features of this disease. We also generated additional lists of DEGs that met different filtering criteria (Supplementary Data 2b, c and 3 for the complete DEG results for each dataset).

To better understand the underlying biological functions and molecular mechanisms associated with the observed DEGs, we performed a hypergeometric test to detect statistically significant overrepresented Gene Ontology (GO) terms ${ }^{24}$ among the DEGs having an absolute Log2FC $>1$ in each dataset separately ${ }^{24}$.

Consistent with the findings of Blanco-Melo et al. ${ }^{22}$, GO enrichment analysis returned terms associated with immune system processes, response to cytokine, stress and viruses, and phosphatidylinositol 3-kinase (PI3K)/AKT signaling pathway, among others (see Supplementary Data 4 for complete results). In addition, we report $285 \mathrm{GO}$ terms common to at least two cell line datasets infected with SARS-CoV-2 and absent in the response to other viruses (Fig. $2 \mathrm{~b}$ and Supplementary Data 5a, b), including neutrophil and granulocyte activation, interleukin-1 (IL-1)-mediated signaling pathway, proteolysis, and stressactivated signaling cascades. We also detected 397 cell linespecific GO terms (76 in NHBE, 160 in A549, and 161 in Calu-3), which were not detected in the other viral datasets (Supplementary Data 5c). Our results show that each cell type regulates specific responses against SARS-CoV-2: A549-specific terms included vacuolar organization, endosome membrane, and protein export, whereas Calu-3-specific terms included oxidative phosphorylation, mitochondria, and cellular response to oxidative stress; NHBE cells had the majority of significant terms involved in cell chemotaxis and leukocyte-mediated immunity. One possible reason for these cell-specific responses is that each cell type expresses different levels of the viral receptor ACE2 (Supplementary Fig. 1).

Next, we wanted to pinpoint intracellular signaling pathways that may be modulated specifically during SARS-CoV-2 infection. A robust bootstrap-based signaling pathway impact analysis (SPIA) enabled us to identify 30 pathways, including many involved in the host immune response, which are significantly enriched among DEGs in at least one virus-infected cell line dataset (Supplementary Data 6). More importantly, we predicted four pathways to be specific to SARS-CoV-2 infection and observed that the significant pathways differ by cell type and multiplicity of infection (MOI). The significant results included only one term common to A549 (MOI 0.2 ) and Calu- 3 cells (MOI 2 ), namely IFN- $\alpha / \beta$ signaling. In addition, we found the amoebiasis pathways (A549 cells, MOI 0.2) and the p75(NTR)-mediated and trka receptor signaling pathways (A549 cells, MOI 2) to be significantly impacted.

We also used a classic hypergeometric method as a complementary approach to our SPIA pathway enrichment analysis. Although there were generally higher numbers of significant results using this method, we observed that the vast majority of enriched terms (false discovery rate $(\mathrm{FDR})<0.05$ ) described infections with various pathogens, innate immunity, metabolism, and cell cycle regulation (Supplementary Data 6). Interestingly, we were able to detect enriched Kyoto Encyclopedia of Genes and Genomes (KEGG) pathways common to at least two SARS-CoV-2-infected cell types and absent from the other virus-infected datasets (Fig. 2b). These included pathways related to infection, cell cycle, endocytosis, signaling pathways, cancer, and other diseases.

Our analyses highlight biological pathways in human lung cells that are altered specifically by SARS-CoV-2 infection, either in a cell-type-specific manner or consistent across cell types. This complements studies identifying pathway-level alterations in immune cells of COVID-19 patients ${ }^{25-27}$.

SARS-CoV-2 infection results in altered lipid-related metabolic fluxes. To better understand how gene expression changes in response to virus infection impact human metabolism, we projected 


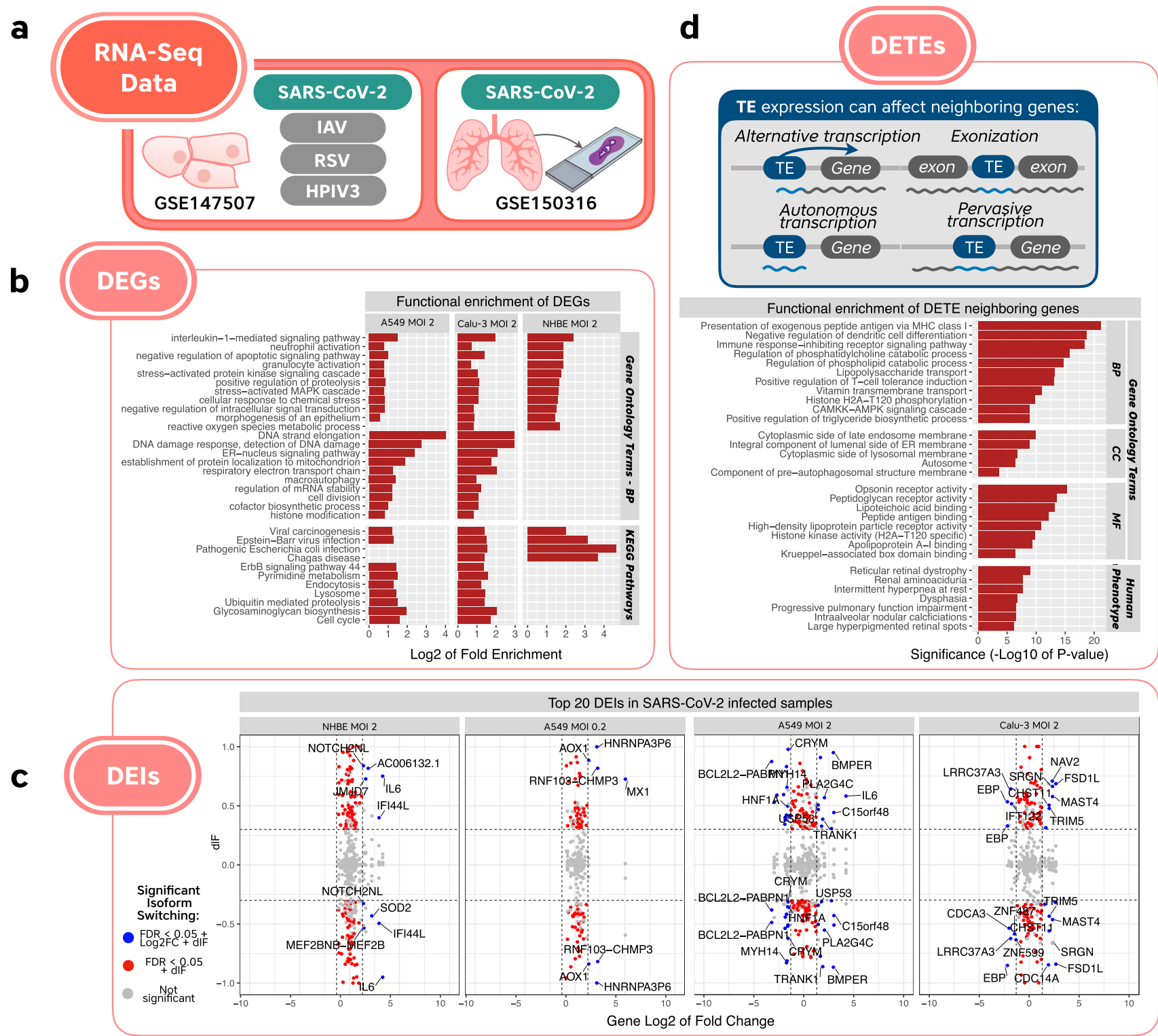

Fig. 2 Overview of the RNA-seq-based results specific to SARS-CoV-2, which were not detected in the other viral infections (IAV, HPIV3, and RSV). a Representation of the RNA-seq studies used in our analyses. b A subset of non-redundant reduced terms consistently enriched in more than one SARSCoV-2 cell line, which were not detected in the other viruses' datasets. c Top 20 differentially expressed isoforms (DEls) in SARS-CoV-2-infected samples. The $y$-axis denotes the differential usage of isoforms between conditions (i.e., difference in isoform fraction, dIF), whereas the $x$-axis represents the overall $\log 2 \mathrm{FC}$ of the corresponding gene. Thus, DEls also detected as differentially expressed genes (DEGs) by this analysis are depicted in blue. $\mathbf{d}$ Different manners by which transposable element (TE) family overexpression might be detected. Although TEs may be autonomously expressed, the old age of most TEs detected points toward either being part of a gene (exonization or alternative promoter) or a result of pervasive transcription. We report the functional enrichment for neighboring genes of differentially expressed TEs (DETEs) specifically upregulated in SARS-CoV-2 Calu-3 and A549 cells (MOI 2). Source data for Fig. 2 is provided in Supplementary Data 18.

the transcriptomic data onto the human metabolic network ${ }^{28}$. This is an important analysis, as it can recover pathway-level changes that are not evident from analyzing dysregulated genes separately. This is based on the fact that the regulation of the entire metabolic pathways can be achieved by targeting few key enzymes via different regulatory mechanisms ${ }^{29-31}$. By integrating information of the metabolic network with differential expression, we can predict which connected pathways were most likely increased or decreased in viral infection $^{32}$.

This analysis detected decreased fluxes in inositol phosphate metabolism in both A549 and Calu-3 cells infected with SARSCoV-2 with an MOI of 2 (Supplementary Data 7). In addition, we detected an increased flux common to A549 and Calu-3 cell lines in reactive oxygen species detoxification, in accordance with previous terms recovered from functional enrichment analyses. Our analysis in A549 cells (MOI 2) also recovered decreased fluxes in several lipid pathways: fatty acid, cholesterol, sphingolipid, and glycerophospholipid, which have been shown as essential for the infection of multiple coronaviruses ${ }^{33}$. Overall, we were able to predict pathway-level changes that were not evident based only on DEGs, given that the control of key enzymes can be enough for the regulation of entire pathways.

SARS-CoV-2 infection induced an isoform switch of genes associated with immunity and mRNA processing. We analyzed 


\begin{tabular}{|c|c|c|c|c|c|}
\hline \multirow[t]{2}{*}{ Gene } & \multicolumn{4}{|c|}{ Cell type and MOI } & \multirow{2}{*}{$\begin{array}{l}\text { Also } \\
\text { detected in } \\
\text { biopsies }\end{array}$} \\
\hline & $\begin{array}{l}\text { A549 } \\
\text { MOI } 0.2\end{array}$ & $\begin{array}{l}\text { A549 } \\
\text { MOI } 2 \\
\end{array}$ & Calu-3 & NHBE & \\
\hline VNN2 & & 6.18 & 0.42 & 6.13 & \\
\hline CSF2 & & 3.56 & 7.30 & 2.70 & \\
\hline WNT7A & & 4.99 & 0.79 & 0.45 & \\
\hline PDZK1IP1 & & 1.72 & 0.70 & 2.28 & \\
\hline SERPINA3 & 0.49 & 1.39 & 0.77 & 1.44 & Case 9 \\
\hline RHCG & & 1.51 & 2.02 & 1.33 & \\
\hline IL32 & & 1.64 & 1.23 & 1.21 & Case 1 \\
\hline PDGFB & & 1.91 & 1.75 & 1.00 & \\
\hline ALDH1A3 & & 1.09 & 1.32 & 0.39 & \\
\hline TLR2 & & 1.63 & 0.89 & 0.84 & \\
\hline SERPINB1 & & 0.61 & 1.17 & 0.72 & \\
\hline PRDM1 & & 0.82 & 3.49 & 0.59 & \\
\hline$M T-T N$ & & 0.55 & 1.70 & 0.33 & \\
\hline ATF4 & & 0.79 & 1.07 & 0.26 & \\
\hline PTPN12 & 0.48 & 0.97 & 1.23 & & \\
\hline DUSP16 & 0.33 & 0.41 & 1.43 & & \\
\hline FKBP5 & & -0.39 & & -0.36 & $\begin{array}{l}\text { Cases } 1 \\
3,8,11\end{array}$ \\
\hline$D A P$ & & -0.18 & -0.61 & & Case 1 \\
\hline $\mathrm{FECH}$ & & -0.27 & -0.36 & & Case 1 \\
\hline$M T-C Y B$ & & -0.30 & -0.26 & & Case 1,8 \\
\hline EIF4A1 & & -0.33 & -0.63 & & Case 1 \\
\hline POLE4 & -0.23 & -0.82 & -1.24 & & \\
\hline DDX39A & -0.23 & -1.27 & -0.54 & & \\
\hline CENPP & & -0.36 & -0.40 & -0.38 & \\
\hline TMEM5OB & & -0.48 & -0.59 & -0.53 & \\
\hline HPS1 & -0.28 & -0.31 & -0.62 & & \\
\hline SNX8 & -0.30 & -0.43 & -0.56 & & \\
\hline
\end{tabular}

Log2 fold change for selected genes that showed significant up- or downregulation in SARSCoV-2-infected samples (FDR-adjusted $p$-value $<0.05$ ) and not in samples infected with the other viruses tested.

$\mathrm{MOI}$ multiplicity of infection.

changes in transcript isoform expression and usage associated with SARS-CoV-2 infection, and predicted whether these changes might result in altered protein function.

We calculated isoform fraction (IF) values as the percentage of an individual isoform's expression level relative to all other isoforms present within the parent gene's expression level as presented in Eq. (1):

$\mathrm{IF}_{\text {isoform } 1}=\frac{\text { Isoform expression } 1}{\text { Gene expression(Isoform expression } 1+\text { isoform } 2+\ldots+\text { isoform } n \text { ) }}$

Differential isoform usage (difference in IF, dIF) is defined as the difference in the fraction of an isoform present between two conditions presented in Eq. (2):

$$
\mathrm{dIF}=\mathrm{IF}_{\text {condition 2 }}-\mathrm{IF}_{\text {condition } 1}
$$

We identified isoforms experiencing a switch in usage $\geq 30 \%$ in absolute value $(\mathrm{dIF} \geq|0.3|)$ across conditions and retrieved those with an FDR-adjusted $p$-value $(q$-value $)<0.05$. Based on these criteria, we detected 3569 differentially expressed isoforms across all samples (Supplementary Table 1 and Supplementary Data 8). We performed biological consequence enrichment analysis to assess whether a particular consequence occurs more frequently than its opposite between conditions (Supplementary Fig. 2). For example, isoforms from A549 cells infected with RSV, IAV, and HPIV3 exhibited significant increases in nonsense-mediated decay
(NMD) sensitivity and intron retention gain, while simultaneously exhibiting decreases in open reading frames (ORFs) and domains present. These conditions also displayed significant changes in splicing patterns, ranging from loss of exon skipping events, changes in usage of alternative transcription start and termination sites, and decreased alternative $5^{\prime}$ - and $3^{\prime}$-splice sites (Supplementary Fig. 3).

In contrast, isoforms from SARS-CoV-2-infected samples displayed no significant global enrichment of biological consequences or alternative splicing events (Supplementary Figs. 2 and 3 , respectively). However, nonsignificant trends (FDRadjusted $p$-value $>0.05$ ) indicated that certain transcripts in SARS-CoV-2 samples experienced decreases in ORF length, numbers of domains, coding capability, intron retention, and NMD (Supplementary Fig. 2). Although these trends were not significant on the genome-wide scale, they implicate that SARSCoV-2 may trigger host machinery to target and aberrantly splice specific isoforms, leading to decreases in transcript length and, therefore, production of truncated proteins or alternative proteins.

To identify the specific isoforms affected by SARS-CoV-2 infection, we analyzed gene expression and isoform usage of individual isoforms in SARS-CoV-2 samples vs. controls. Results showed significant changes in gene expression and isoform usage at the individual gene level, with top-expressing isoforms associated with genes encoding cellular processes such as immune response and antiviral activity (IFI44L, IL-6, MX1, TRIM5), transcription and mRNA processing (DDX10, HNRNPA3F6, JMJD7, ZNF487, ZNF599), and cell cycle and survival (BCL2L2$P A B P N 1, C D C A 3)$ (Fig. 2c, Supplementary Fig. 4, and Supplementary Data 8).

We noticed that $I L-6$, a gene encoding a cytokine involved in acute and chronic inflammatory responses, displayed significant changes in both gene expression and isoform usage in SARSCoV-2 infection. IL-6 expression increased by two- to sixfold with an MOI of 2 (Fig. 3b). To date, the Ensembl Genome Reference Consortium has identified nine $I L-6$ isoforms in humans, with the traditional transcript having six exons (IL6-204), five of which contain coding elements (Fig. 3a). NHBE cells expressed four known IL-6 isoforms, whereas A549 cells expressed one unknown and six known isoforms (Supplementary Fig. 5). When evaluating the actual isoforms used across conditions, SARS-CoV-2-infected NHBE cells used three out of four isoforms observed, whereas SARS-CoV-2-infected A549 cells used all seven observed isoforms. For example, in the case of NHBE SARS-CoV-2 samples, the IF for the IL6-201 isoform $=0.75, I L 6-204=0.05, I L 6-206=$ 0.09 , and $I L 6-209=0.06$, and the sum of these IF values $=0.95$, or $95 \%$ usage of the $I L-6$ gene relative to mock. SARS-CoV-2 samples (A549 MOI 0.2, A549 MOI 2, and NHBE MOI 2) exhibited exclusive usage of non-canonical isoform IL6-201 (Fig. 3c) and, inversely, mock samples almost exclusively utilized the IL6-204 transcript. In NHBE-infected cells, isoform IL6-201 experienced a significant increase in usage $(\mathrm{dIF}=0.75)$ and IL6204 a significant decrease in usage $(\mathrm{dIF}=-0.95)$ when compared to mock conditions. Similarly, isoform IL6-201 in A549-infected cells experienced an increase in usage $(\mathrm{dIF}=0.58)$, whereas uses of all other isoforms remained nonsignificant in comparison to mock conditions.

The IL6-201 and IL6-204 isoforms both contain five coding exons, and according to Ensembl, both are predicted to produce the same 212 amino acid protein product. The main difference between both isoforms is that IL6-201 does not contain exon 1 ( $5^{\prime}$-untranslated region, $5^{\prime}$-UTR), which is present in IL6-204. The $5^{\prime}$-UTRs are traditionally involved in translational regulation, either promoting or inhibiting translation, depending upon the sequence and secondary RNA structure ${ }^{34,35}$ or modulating 
a

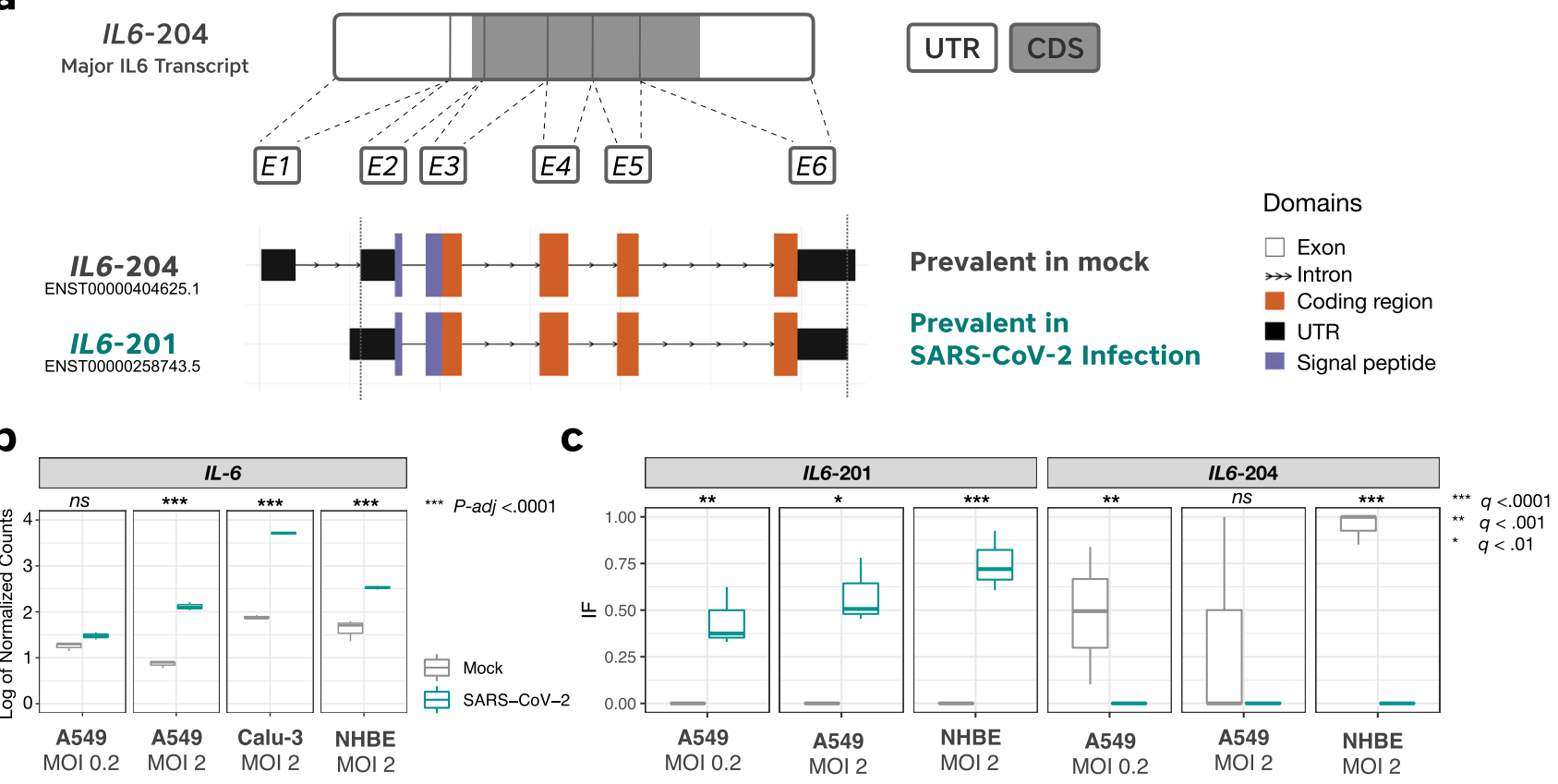

Fig. 3 Isoform usage of IL-6 transcripts in SARS-CoV-2-infected cells. a IL6-204 is the major IL-6 transcript and is composed of 6 exons, five (E2, E3, E4, E5, E6) containing coding sequences (CDS) and one (E1) containing exclusively a 5'-untranslated region (5'-UTR). Both isoforms (IL6-204 and IL6-201) have the same protein-coding capability. The main difference between them is the absence of E1 in IL6-201, which is the major induced isoform upon SARSCoV-2 infection. b Gene expression of IL-6 in all SARS-CoV-2 cell line samples (A549 multiplicity of infection (MOI) 0.2 and 2; Calu-3 and NHBE MOI 2). Each boxplot represents three biological replicates and statistical testing was performed with DESeq2 (detailed in "Methods" section). Exact $p$-values are available in Supplementary Data 2. c Isoform usage switch between both isoforms in SARS-CoV-2-infected cell line samples. This figure shows that IL6-204 is almost exclusively expressed in uninfected (mock) cells, whereas IL6-201 is almost exclusively expressed in SARS-CoV-2-infected cells. Each boxplot represents three biological replicates and statistical testing was performed with IsoformSwitchAnalyzeR and exact $q$-values are available in Supplementary Data 8. Source data for Fig. 3 is provided in Supplementary Data 18.

mRNA stability ${ }^{36}$. Thus, this isoform switch may be a mechanism to regulate IL-6 protein synthesis through control of translation rate and/or mRNA stability.

Overexpression of TE families close to immune-associated genes upon SARS-CoV-2 infection. TEs are repeated DNA sequences that are able to spread across the genome, representing around two-thirds of the human genetic material ${ }^{37}$. TEs can be grouped into two different classes regarding their transposition mechanisms: (1) DNA elements, which are mobilized via a DNA molecule and make up around $\sim 3 \%$ of the human genome $e^{38}$, and (2) retrotransposons, which have an RNA intermediate. Retrotransposons can be further divided into long-term repeat (LTR) elements, also named endogenous retroviruses, which account for $\sim 8 \%$ of the human genome, or long and short interspersed nuclear elements (LINEs and SINEs) and SINE-VNTR-Alu elements, which lack LTRs and are the most abundant superfamilies in the human genome, accounting for around one-third of DNA sequences ${ }^{38}$. Although the human genome is bursting with TEs, most TE families are unable to transpose, either because they lost their transpositional machinery or because they have accumulated mutations that hinder their activity. There are only three TE families currently active in the human genome: LINE1, Alu (SINE) subfamily, and SVAs ${ }^{39}$. Nevertheless, the graveyard of dead TEs in the human genome has been repeatedly shown to regulate host gene expression, thus participating in key developmental and immune networks ${ }^{40-42}$. Therefore, searching for TE deregulation upon viral infection might shed light into activation of young TE families, but also pinpoint changes in gene regulatory networks.
To estimate the expression of TE families and their possible roles in SARS-CoV-2 infection, we mapped the RNA-seq reads against all annotated TE human families (see "Methods" section) and detected differentially expressed TE (DETE) families (Fig. 2d and Supplementary Data 9). We found 68 common TE families upregulated in SARS-CoV-2-infected A549 and Calu-3 cells (MOI 2), including 53 retrotransposons (24 LINEs, 27 LTRs, and two SINEs). It is important to note that none of the current transpositionally active human TE families were found to be upregulated in SARS-CoV-2-infected cells. From this list, we excluded all TE families detected in cells infected with the other viruses. This allowed us to identify 16 families that were specifically upregulated in Calu-3 and A549 cells infected with SARS-CoV-2, and not in the other viral infections. The 16 families identified are MER77B, MamRep4096, MLT2C2, PABL_A, Charlie9, MER34A, L1MEg1, LTR13A, L1MB5, MER11C, MER41B, LTR79, THE1D-int, MLT1I, MLT1F1, and MamRep137. Most of the TE families uncovered are ancient elements, and none are capable of transposing $43-45$. Eleven of the 16 TE families specifically upregulated in SARS-CoV-2-infected cells are LTR elements and include well-known TE immune regulators. For instance, MER41B (primate-specific TE family) is known to contribute to IFN- $\gamma$-inducible binding sites (bound by STAT1 and/or IRF1 $)^{46,47}$. Other LTR elements are also enriched in STAT1-binding sites (MER77B, LTR13, and MLT11) ${ }^{46}$ or have been shown to act as cellular gene enhancers (LTR13A ${ }^{48,49}$ ).

In humans, TEs have been shown to accumulate in mammalian-specific gene regulatory sequences, such as within immunity-related gene transcripts ${ }^{50}$. Given that at least four TE families identified are well-known host-gene regulators, along with the general ability of TE families to impact nearby gene 
expression, we further investigated the functional enrichment of genes near these upregulated TE families (Supplementary Data 10). The GREAT method used for this analysis extends the regulatory domain of each annotated gene to $5 \mathrm{~kb}$ upstream and $1 \mathrm{~kb}$ downstream the transcription start site, as we still lack precise maps of gene regulatory regions ${ }^{51}$. We detected GO functional enrichment of several immunity-related terms (e.g., major histocompatibility complex (MHC) protein complex, antigen processing, regulation of dendritic cell differentiation, and T-cell tolerance induction), metabolism-related terms (such as regulation of phospholipid catabolic process), and, interestingly, a specific human phenotype term called "progressive pulmonary function impairment" (Fig. 2d).

Even though we did not limit our search only to neighboring genes which were also DE, we found several similar (and very specific) enriched terms in both analyses, for instance, related to endosomes, endoplasmic reticulum, and vitamin (cofactor) metabolism, among others. This result supports the idea that some responses during infection could be related to TE-mediated transcriptional regulation. Finally, when we searched for enriched terms related to each one of the 16 families separately, we also detected immunity-related enriched terms such as regulation of ILs, antigen processing, TGF- $\beta$ receptor binding, and temperature homeostasis. It is important to note that given the old age of some of the TEs detected, overexpression might be associated with pervasive transcription or inclusion of TE copies within unspliced introns (Fig. 2d). In conclusion, we were able to demonstrate that 16 TE families are upregulated specifically upon SARS-CoV-2 infection, including four TE families previously shown to harbor STAT1/IRF1-binding sites, and are enriched close to immunityrelated genes. Finally, to clearly pinpoint if such TE families are responsible for nearby gene regulation, future work should focus on TE-gene chimeric transcript searches (using long read RNAseq or paired-end reads), mapping of regulatory sequences within TE copies using chromatin-related methods such as ATAC-seq, and deletion of TE copies followed by analysis of gene expression.

The SARS-CoV-2 genome is enriched in binding motifs for 40 human proteins, most of them conserved across SARS-CoV-2 isolates. The SARS-CoV-2 virus possesses a positive-sense, single-stranded, monopartite RNA genome. Such viruses are wellknown to co-opt host RBPs for diverse processes including viral replication, translation, viral RNA stability, assembly of viral protein complexes, and regulation of viral protein activity ${ }^{14,15}$. Therefore, we sought to predict host RBPs that may form functionally significant interactions with the SARS-CoV-2 genome.

To do so, we first filtered the AtTRACT database ${ }^{52}$ to obtain a list of 102 human RBPs and 205 associated position weight matrices (PWMs) describing the experimentally determined sequence-binding preferences of these proteins. We then scanned the SARS-CoV-2 reference genome sequence to identify potential binding sites for these proteins. Figure 4 illustrates our analysis schema. We identified 99 human RBPs with a total of 11,897 potential binding sites in the SARS-CoV-2 positive-sense genome (Supplementary Data 11).

As the SARS-CoV-2 genome produces negative-sense intermediates as part of the replication process ${ }^{53}$, we also scanned the negative-sense genome sequence, where we found 11,333 potential binding sites for 96 RBPs (Supplementary Data 11).

To find RBPs whose binding sites occur in the SARS-CoV-2 genome more or less frequently than expected by chance, we repeatedly scrambled the genome sequence to create 1000 simulated genome sequences with an identical nucleotide composition to the SARS-CoV-2 genome sequence (30\% A, $18 \% \mathrm{C}, 20 \% \mathrm{G}$, $32 \% \mathrm{~T})$. We used these 1000 simulated genomes to determine a background distribution of the number of binding sites found for a specific RBP. This allowed us to pinpoint RBPs with significantly more or fewer binding sites in the actual SARSCoV-2 genome than expected based on the background distribution (two-tailed $z$-test, FDR-corrected $P<0.01$ ). To retrieve RBPs whose motifs were enriched in specific genomic regions, we also repeated this analysis independently for the SARS-CoV-2 5'-UTR, 3'-UTR, intergenic regions, and for the negative-sense genome sequence. Motifs for 40 human RBPs were found to be enriched in at least one of the tested genomic regions, whereas motifs for 23 human RBPs were found to be depleted in at least one of the tested regions (Supplementary Data 12). Although experimental validation would be required to confirm the importance of these putative interactions, enrichment or depletion of binding sites for an RBP is suggestive that it may be beneficial or inhibitory, respectively, to viral replication.

We next examined whether any of the 6936 putative binding sites for these 40 enriched RBPs were conserved across SARSCoV-2 isolates. We found that 6591 putative binding sites, representing 34 RBPs, were conserved across more than $95 \%$ of SARS-CoV-2 genome sequences in the GISAID database ( $\geq 171,953$ out of 181,003 genomes). However, this is of limited significance, as the RBP-binding sites in coding regions are likely to be conserved due to evolutionary pressure on protein sequences rather than the RBP-binding ability. We therefore repeated this analysis focusing only on putative RBP-binding sites in the SARS-CoV-2 UTRs and intergenic regions. There were 124 putative RBP-binding sites for 21 enriched RBPs in the UTRs and intergenic regions. Of these, 50 putative RBP-binding sites for 17 RBPs were conserved in $>95 \%$ of the available genome sequences; 6 in the $5^{\prime}$-UTR, 5 in the $3^{\prime}$-UTR, and 39 in intergenic regions (Supplementary Table 2).

Subsequently, we interrogated publicly available data to further investigate the putative SARS-CoV-2/RBP interactions (Supplementary Data 13). According to GTEx data ${ }^{54}, 39$ of the 40 enriched RBPs and all 23 of the depleted RBPs were expressed in human lung tissue. Furthermore, 31 of 40 enriched RBPs and 22 of 23 depleted RBPs were co-expressed with the ACE2 and TMPRSS2 receptor genes in single-cell RNA-seq data from human lung cells ${ }^{55}$, indicating that they are present in cells that are susceptible to SARS-CoV-2 infection. We next checked whether any of these RBPs have been reported to interact with SARS-CoV-2 proteins and found that human poly(A)-binding protein cytoplasmic 1 and 4 (PABPC1 and PABPC4, respectively) were reported to bind to the viral $\mathrm{N}$ protein in a recent study ${ }^{18}$. If this report is correct, these RBPs may interact with both the SARS-CoV-2 RNA and proteins. Finally, we combined these results with our analysis of differential gene expression to identify SARS-CoV-2-interacting RBPs that also show expression changes upon infection. The results of this analysis are summarized for selected RBPs in Table 2. Based on our computational analysis and existing literature, we highlight these enriched RBPs for their potential functional interaction with SARS-CoV-2 RNA.

Motif enrichment in SARS-CoV-2 differs from related coronaviruses. We repeated the above analysis to calculate the enrichment and depletion of RBP-binding motifs in the genomes of two related coronaviruses: (i) the SARS-CoV virus that caused the SARS outbreak in 2002-2003 and (ii) RaTG13, a bat coronavirus with a genome that is $96 \%$ identical with that of SARS$\mathrm{CoV}-2^{2,6}$. We found that the pattern of enrichment and depletion of RBP-binding motifs in SARS-CoV-2 is different from that of the other two viruses (Supplementary Data 14 and 15). Specifically, the SARS-CoV-2 genome is uniquely enriched for binding sites of CELF5 in its $5^{\prime}$-UTR, PPIE in its $3^{\prime}$-UTR, and ELAVL1 in 


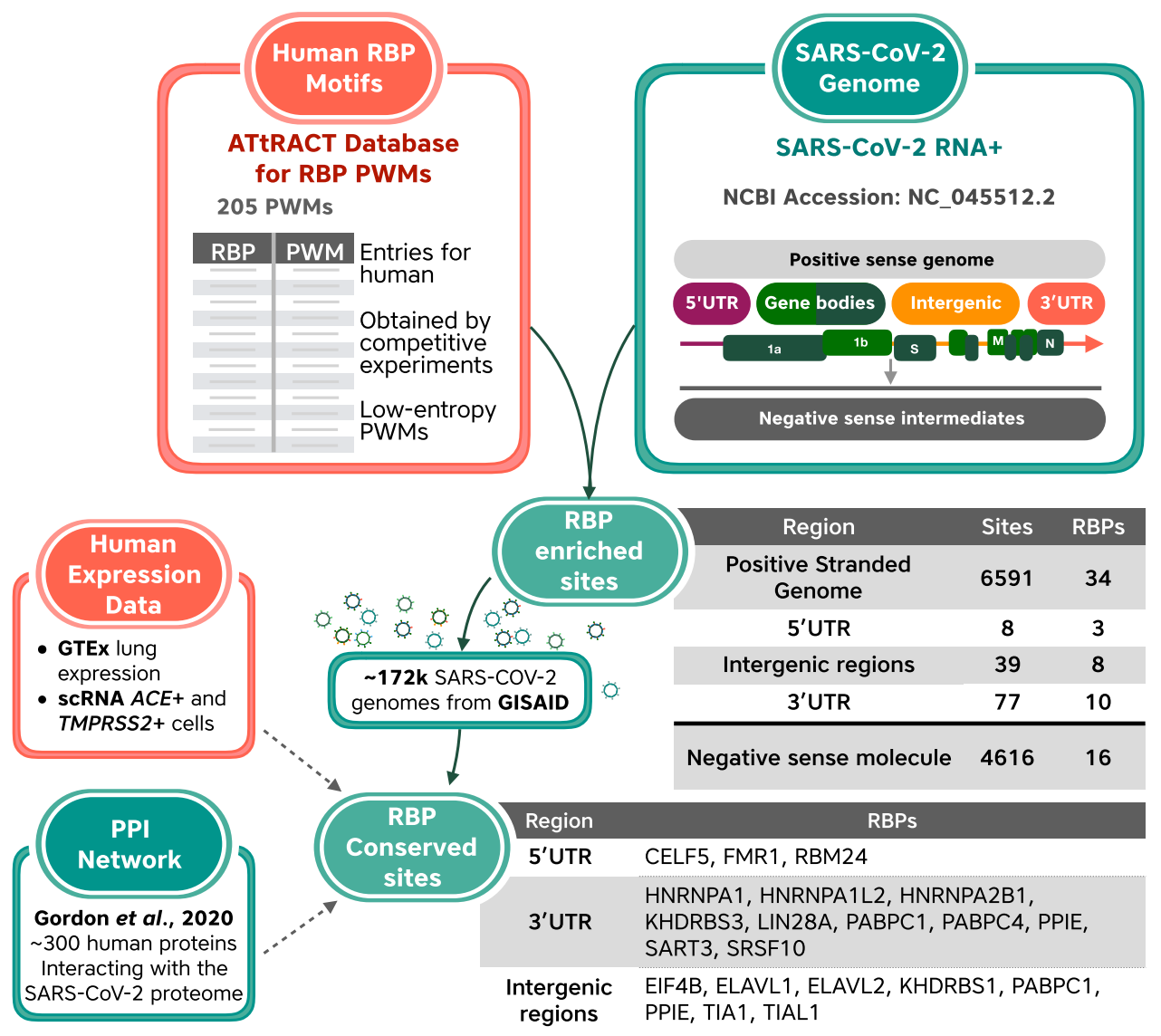

Fig. 4 Workflow and selected results for the analysis of potential binding sites for human RNA-binding proteins (RBPs) in the SARS-CoV-2 genome. In orange, human RNA-binding protein (RBP) position weight matrices (PWMs) from the ATtRACT database were used as input to search for putative binding sites in the SARS-CoV-2 virus genome (green). Binding motifs of several RBPs were detected to be enriched/depleted within the positive-strand genome (containing genes, 5'- and 3'-untranslated regions (UTRs), and intergenic regions) and the negative-sense intermediates. Conserved RBP-binding sites were determined from the multiple sequence alignment of $\sim 180 \mathrm{k}$ SARS-CoV-2 genomes available from GISAID. Finally, we included information from human gene expression data and protein-protein interaction networks for human and SARS-CoV-2 that are publicly available.

the viral negative-sense RNA molecule. Interestingly, ELAVL1 is a known stabilizer of RNA ${ }^{56}$, whereas CELF5 and PPIE participate in splicing 57,58 . Despite the high sequence identity between the two genomes, the single binding site for CELF5 on the SARSCoV-2 $5^{\prime}$-UTR is conserved in $95.8 \%$ of available SARS-CoV-2 genome sequences but absent in the $5^{\prime}$-UTR of RaTG13.

A viral genome variant associated with host age. We used the meta-CATS software ${ }^{59}$ to test whether any viral sequence variants were associated with a change in disease severity, age, or biological sex in human hosts. We computed statistical correlations between 8079 complete SARS-CoV-2 genomes and the associated clinical metadata for each genome (e.g., severe, moderate, or mild disease; decade of age; and male or female). Briefly, this process calculates a $\chi^{2}$-score from a contingency table that contains the nucleotides present at each aligned position and the clinical metadata. The resulting $p$-value identifies positions that contain a statistically significant skew in the distribution of bases between the metadata categories. The alignment consisted of 30,649 nucleotide positions and 28,870 of these aligned positions contained at least one variant. We identified 3960 positions that contained at least one significant pairwise correlation with disease severity, 25 with patient age, and 883 with biological sex.

The FDR-corrected statistical results from this $\chi^{2}$-test of independence revealed a large number of nucleotide positions that showed statistically significant skew in the distribution of bases (Supplementary Data 16). However, further analysis revealed a low specificity for the vast majority of these results due primarily to the presence of ambiguous bases in a small number of the consensus genomes. This indicates that disease severity or infection of either biological sex of the patient cannot be solely attributed to a single viral variant.

In contrast, we identified a significant and specific skew in the distribution of bases between host age and aligned position 14,525 (cytosine to thymine at unaligned position 14,408 in the reference genome). The CCT to CTT codon variation (P323L in the RNA-dependent RNA polymerase (RdRP)) was found to significantly differ only when patients between 20 and 30 years old were compared against patients who were at least 85 years old ( $p$-value $<0.04)$.

The distribution of bases between these two populations were $246 \mathrm{C}, 760 \mathrm{~T}$, and $3 \mathrm{Y}$ for the patients in their $20 \mathrm{~s}$, whereas the distribution for patients older than 85 years was 27C, 249T, and $7 \mathrm{Y}$. These results show a $1: 3$ ratio of $\mathrm{C}$ to $\mathrm{T}$ in young patients and a ratio of $\sim 1: 9$ of $\mathrm{C}$ to $\mathrm{T}$ in older patients. Two additional Pearson's $\chi^{2}$-tests were subsequently performed, to account for the biological sex of the patients as covariates with age. These showed significant skew in the distribution of bases from viruses infecting 20 - to 29-year-old males vs. $>85$-year-old males 
Table 2 RNA-binding proteins (RBPs) predicted to interact with SARS-CoV-2.

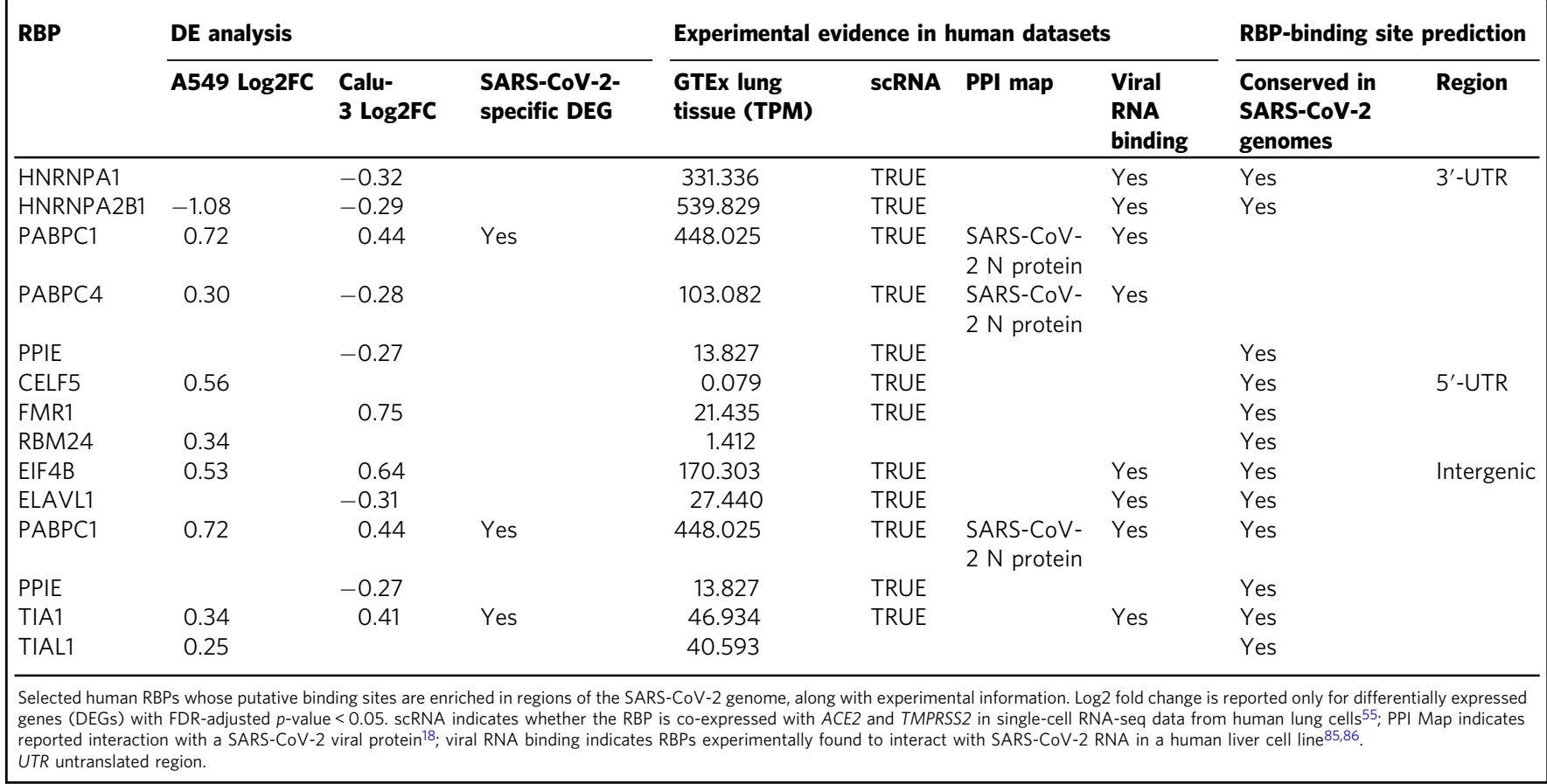

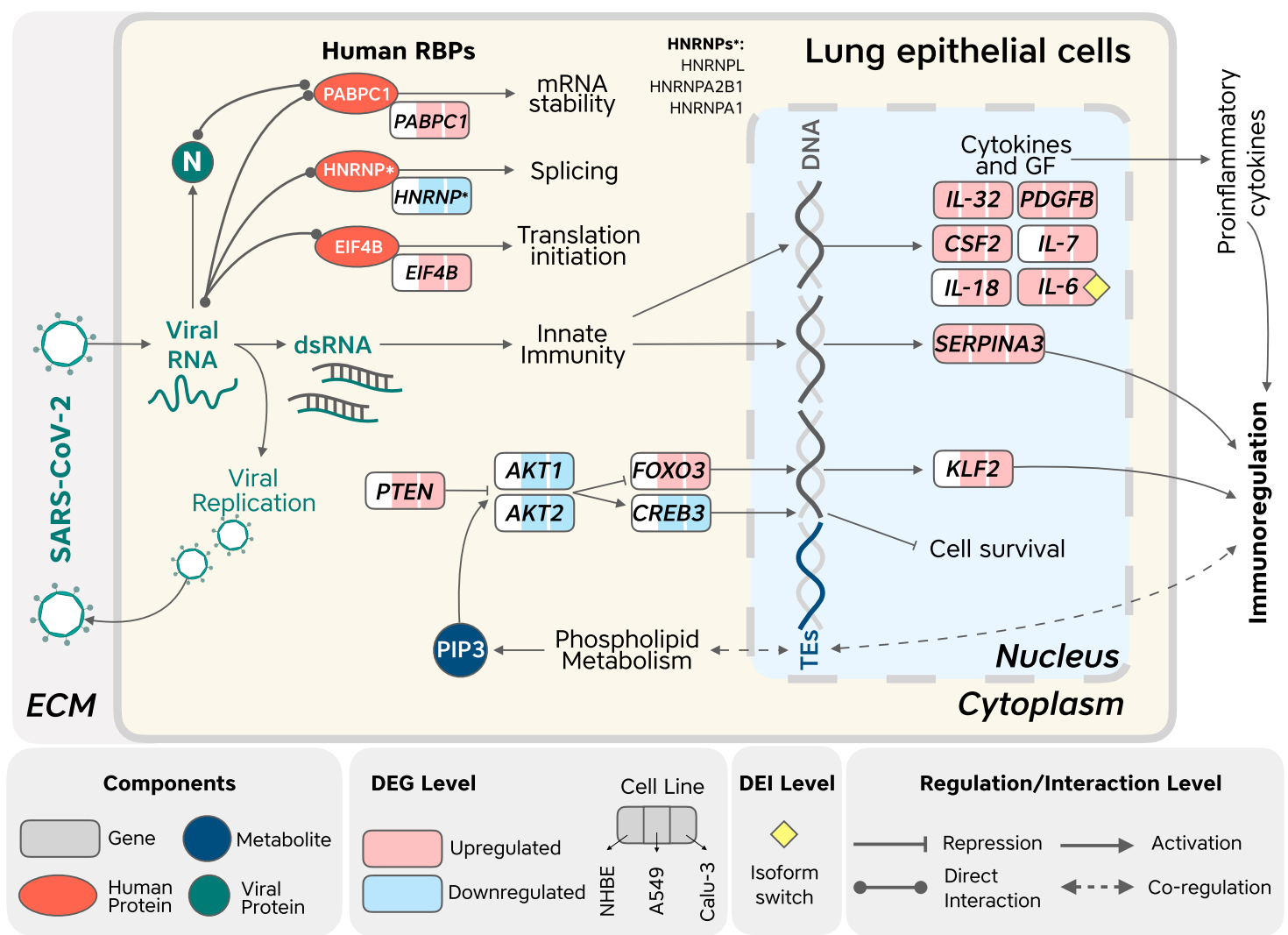

Fig. 5 Overview of human factors specific to SARS-CoV-2 infection detected by our analyses. This figure includes human RNA-binding proteins (RBPs), whose binding sites are enriched and conserved in the SARS-CoV-2 genome but not in the genomes of related viruses, and gene isoforms and metabolites that are consistently altered in response to SARS-CoV-2 infection of lung epithelial cells but not in infection with the other tested viruses. ECM: extracellular matrix. 
$\left(p\right.$-values $\left.=2.2 \times 10^{-16}\right)$, as well as in 20 - to 29 -year-old females vs. $>85$-year-old females $\left(p\right.$-value $\left.=3.2 \times 10^{-7}\right)$.

\section{Discussion}

Here we report the results of a complementary panel of analyses that, together, enable a better understanding of host-pathogen interactions, which contribute to SARS-CoV-2 replication and pathogenesis in the human respiratory system. Figure 5 illustrates an overview of interesting host and viral factors detected in this work.

We performed a cross-dataset analysis of differential gene expression, highlighting specific genes that may play a role in the unique pathogenic features of COVID-19. Moreover, this analysis formed the basis for our subsequent dissection of pathway activity, metabolism, TEs, and regulatory activity in COVID-19. We discovered several known immunoregulators among the genes specifically and consistently altered in response to SARS$\mathrm{CoV}-2$, which points to distinct features of the immune response to this pathogen.

For example, CSF2, which encodes the granulocytemacrophage colony stimulating factor (GM-CSF), was among the most highly upregulated genes in SARS-CoV-2-infected cells and is associated with tissue hyper-inflammation ${ }^{60}$. GM-CSF induces survival and activation in macrophages and neutrophils, and has been found at high levels in the blood of severe COVID19 patients $^{61}$, and several clinical trials are planned using agents that target GM-CSF or its receptor ${ }^{62}$. Another proinflammatory cytokine specifically upregulated in SARS-CoV-2-infected cells is IL32, which together with GM-CSF, promotes the release of tumor necrosis factor and IL- 6 in a positive loop, and contributes to the cytokine storm ${ }^{63}$. In accordance, $I L-6$ was upregulated in the three SARS-CoV-2-infected cell lines analyzed here. Moreover, not only upregulation but also a shift in isoform usage of $I L$ 6 was detected in three SARS-CoV-2-infected datasets. A shift in $5^{\prime}$-UTR usage in the presence of SARS-CoV-2 may be attributed to indirect host cell signaling cascades that trigger changes in transcription and splicing activity, which could also explain the overall increase in $I L-6$ expression.

Aberrant isoform usage and splicing have previously been associated with the human antiviral response, cancer, and neurodegenerative diseases ${ }^{64-66}$; moreover, a recent study found that SARS-CoV-2 infection alters isoform usage of the ERAP2 gene ${ }^{67}$. However, to our knowledge, ours is the first genome-wide analysis of the effect of SARS-CoV-2 on isoform usage. Additional experiments are needed to validate the effect of the IL6-201 isoform on IL-6 protein activity in SARS-CoV-2-infected lung tissues. However, elevated IL-6 was observed in more than half of COVID-19 patients ${ }^{68}$ and was associated with COVID-19 complications, progression and poor prognosis, respiratory failure, sepsis, and mortality risk ${ }^{69-73}$. Our observations suggest the possibility that isoform switching, as well as upregulation of gene expression, may contribute to this IL-6 elevation. Although clinical trials evaluating IL-6 inhibitors in immune-based therapies exist ${ }^{74}$, the value in using IL- 6 activity, and by extension all relevant isoforms, as a prognostic tool in determining severity and disease progression in SARS-CoV-2-infected patients is apparent.

SERPINA3, an essential enzyme in the regulation of leukocyte proteases, is induced by cytokines ${ }^{75}$ and has been proposed to inhibit viral replication ${ }^{23}$. This was the only gene consistently upregulated in all cell line samples infected with SARS-CoV-2 and absent from the other virus-infected datasets in this study. The VNN2 gene was also upregulated in our analysis. Vanins are involved in proinflammatory and oxidative processes, and VNN2 plays a role in neutrophil migration by regulating b2 integrin ${ }^{76}$.
Downregulated genes included SNX8, which has been reported in RNA virus-triggered induction of antiviral genes ${ }^{23,77}$, and FKBP5, a regulator of NF- $\kappa B$ activity ${ }^{78}$. These results suggest that SARSCoV-2 tends to indirectly target specific genes involved in genome replication and host antiviral immune response without eliciting a global change in cellular transcript processing or protein production.

One of the first and most important innate antiviral responses is the production of type I IFN. This induces hundreds of IFNstimulated genes, which limit virus spread and infection. Expression of SARS-CoV-2 proteins has previously been reported to inhibit the type I IFN signaling pathway ${ }^{79}$. Our signaling pathway analysis supported this by showing that type I IFN response was greatly impacted upon SARS-CoV-2 infection. We also observed elevated expression of PRDM1 (Blimp-1) in SARSCoV-2-infected cells, which may contribute to the critical regulation of IFN signaling cascades. Interestingly, the TE family LTR13, which was also upregulated, is enriched in PRDM1 binding sites ${ }^{80}$. Therefore, it is possible that regulatory factors involved in IFN and immune response in SARS-CoV-2 infection could be partially attributed to TE transcriptional activation. Similarly, we detected upregulation of several immunoregulatory TE families in SARS-CoV-2-infected cells. The MER41B family, for instance, is known to contribute to IFN- $\gamma$ inducible binding sites (bound by STAT1 and/or IRF1). Functional enrichment of nearby genes was in accordance with these findings, as several immunity-related terms were enriched along with "progressive pulmonary impairment."

In parallel, TEs seem to be co-regulated with phospholipid metabolism, which directly affects the PI3K/AKT signaling pathway, central to the immune response. Alterations in phospholipid metabolism and the PI3K/AKT pathway were detected in our metabolic flux analysis and functional enrichment analysis, respectively. A recent screen for host genes required for SARSCoV-2 infection identified three members from the PI3K pathway ${ }^{19}$. In addition, phosphatidylinositol metabolic processes are important for the infection of multiple coronaviruses ${ }^{33}$ and it is well-known that lipid metabolism is essential throughout the life cycle of several viruses ${ }^{81,82}$. Moreover, both glycerophospholipids and fatty acids were reported to be significantly dysregulated in COVID-19 patients ${ }^{83}$. Finally, alteration of fatty acid metabolites in COVID-19 patients was highly correlated with IL6 levels ${ }^{84}$, showing the potential of genome-wide complementary approaches to better understand this complex disease.

RBPs are likely candidates for host factors involved in the response of human cells to SARS-CoV-2, as well as viral manipulation of host machinery. During preparation of this study, two experimental studies ${ }^{85,86}$ reported hundreds of proteins that interact with SARS-CoV-2 RNA in human liver-derived cell lines. Encouragingly, they validated binding of several candidate proteins highlighted by our analysis (Table 2 and Supplementary Data 13). However, they did not identify the specific sites where these proteins bind to viral RNA and a deeper understanding of which RBPs promote or inhibit viral activity remains necessary. Our analysis complements these studies by (1) identifying putative binding sites for each protein on the viral genome, (2) identifying proteins whose binding sites were significantly enriched or depleted in the viral genome, and (3) identifying potential binding sites that are conserved and specific to SARS-CoV-2. We suggest that these proteins are likely to include functionally important interactions and should be the focus of experimental studies.

One of the RBPs whose potential binding sites are enriched and conserved in the SARS-CoV-2 genome is eIF4b, suggesting that SARS-CoV-2 viral protein translation could be eIF4b dependent. We also detected upregulation of the EIF4B gene in A549 and 
Calu-3 cells, which might indicate that this protein is sequestered by the virus and, therefore, cells need to increase its production. Another conserved RBP, which was also upregulated in infected cells, is the PABPC1, which is involved in mRNA stability and translation. PABPC1 has been implicated in multiple viral infections; it is modulated to inhibit host protein translation, promoting viral RNA access to the host translational machinery ${ }^{87}$. Interestingly, PABPC1 and PABPC4 have been reported to interact with the SARS-CoV-2 N protein, which stabilizes the viral genome ${ }^{18}$. This raises the possibility that the viral genome, $\mathrm{N}$ protein, and human $\mathrm{PABP}$ proteins may participate in a joint protein-RNA complex that assists in viral genome stability, replication, and/or translation ${ }^{87-91}$.

Binding motifs for hnRNPA1 were enriched specifically in the $3^{\prime}$-UTR of SARS-CoV-2, even though they were depleted in the genome overall. hnRNPA1 interacts with $3^{\prime}$-UTRs of other coronaviruses and participates in transcription and replication of the murine hepatitis virus ${ }^{92-94}$. The hnRNPA1 gene, along with hnRNPA2B1, was downregulated in Calu-3 cells and, in contrast to the previous examples of upregulated genes, could denote a response from human cells to control viral replication.

Finally, we identified a significant association between a viral sequence variant and age of the host. The P323L mutation in the RdRP was previously shown to be associated with changes in geographical location of the viral strain ${ }^{95}$, although not with the age of the patient. It is possible that intracellular characteristics associated with senescence favor one allele in the polymerase over the other, such as stabilizing the interaction between the RdRp and the viral nsp8 proteins ${ }^{96,97}$. Such possibilities are consistent with previous indications that host cellular factors are critical to SARS-CoV-2 sequence evolution ${ }^{98}$. Our statistical analysis may contain sources of bias that are not limited to the number of genome sequences being collected earlier vs. later in the pandemic and the availability of genomes lacking complete clinical metadata. Although we examined patient sex as a possible covariate with age, it is impossible to account for all possible covariates due to the lack of data at the current time. Additional annotated datasets, as well as lab experiments are required to better elucidate the effect(s) of such viral sequence variants on the host response.

In conclusion, we envision that applying this workflow will yield important mechanistic insights in future analyses on emerging pathogens and we provide all source code freely for future use. Similarly, we expect that these findings will give rise to future studies that elucidate the underlying mechanism(s) responsible for such host-pathogen interactions. Modulating the host components of these mechanisms can aid in the selection of host-based drug targets, prophylactics, and/or therapeutics to reduce virus infection and replication with minimal adverse effects in humans.

\section{Methods}

RNA-seq data processing and differential expression analysis. Two datasets were downloaded from the NCBI Gene Expression Omnibus (GEO) database. The first dataset, GSE147507 22 , includes gene expression measurements from three cell lines derived from the human respiratory system (NHBE, A549, Calu-3) infected either with SARS-CoV-2, IAV, RSV, or HPIV3. The second dataset, GSE150316, includes RNA-seq extracted from FFPE histological sections of lung biopsies from COVID-19 deceased patients and healthy individuals. Supplementary Data 1 describes these datasets in detail.

For the first dataset (GSE147507), data were downloaded from Sequence Read Archive (SRA) using sra-tools (v2.10.8; https://github.com/ncbi/sra-tools) and transformed to FASTQ with fastq-dump. FastQC (v0.11.9; https://github.com/sandrews/FastQC) and MultiQC (v1.9) ${ }^{99}$ were employed to assess the quality of the data and the need to trim reads and/or remove adapters. Selected datasets were mapped to the human reference genome (GENCODE Release 19, GRCh37.p13) using STAR (v.2.7.3a) ${ }^{100}$. Alignment statistics were used to determine which datasets should be included in subsequent steps. The resulting SAM files were converted to BAM files employing samtools (v1.9) ${ }^{101}$. Next, read quantification was performed using StringTie (v2.1.1) ${ }^{102}$ and the output data were post-processed with an auxiliary Python script provided by the same developers to produce files ready for subsequent downstream analyses. Expression was quantified for 57,820 genes based on GENCODE Release 19. For the second gene expression dataset (GSE150316), raw counts for 59,091 genes were directly downloaded from GEO.

DESeq2 (v1.26.0) ${ }^{103}$ was used in both cases to identify DEGs. Finally, an exploratory data analysis was carried out based on the transformed values obtained after applying the variance stabilizing transformation ${ }^{104}$ implemented in the vst() function of DESeq2 ${ }^{103}$. Principal component analysis (PCA) was performed on the samples to evaluate the main sources of variation in the data and to remove outlier samples. Based on the PCA plots, no obvious outliers were detected in GSE147507; however, four entire samples (Cases 4, 6, 7, and 10) along with replicate 2 from Case 5 were detected as outliers and discarded from GSE150316 (Supplementary Fig. 6 and Supplementary Data 1).

GO enrichment analysis. The DEGs produced by DESeq 2 with an absolute $\log 2 \mathrm{FC}>1$ and FDR-adjusted $p$-value $<0.05$ were used as input to a general GO enrichment analysis ${ }^{105}$. Each term was subjected to a hypergeometric test from the GOstats package (v2.54.0) ${ }^{106}$ and the $p$-values were corrected for multiple hypothesis testing, employing the Bonferroni method ${ }^{107}$. GO terms with a significant adjusted $p$-value $<0.05$ were reduced to representative non-redundant terms with the use of REVIGO ${ }^{108}$.

Host signaling pathway enrichment. The DEG lists produced by DESeq 2 with an absolute $\log 2 \mathrm{FC}>1$ and FDR-adjusted $p$-value $<0.05$ were used as input to the SPIA algorithm to identify significantly affected pathways from the $\mathrm{R}$ graphite library ${ }^{109,110}$. Pathways with Bonferroni-adjusted $p$-values $<0.05$ were included in downstream analyses. The significant results for all comparisons from publicly available data from KEGG, Reactome, Panther, BioCarta, and NCI were then compiled to facilitate downstream comparison. Hypergeometric pathway enrichments were performed employing the Database for Annotation, Visualization, and Integrated Discovery (DAVID, v6.8) ${ }^{111}$.

\section{Integration of transcriptomic analysis with human metabolic network. To} predict increased or decreased fluxes of reactions, we projected the transcriptomic data onto the human reconstructed metabolic network Recon (v2.2) ${ }^{28}$. This can be done based on the fact that the metabolic network includes gene-protein-reaction associations (GPRs), which in turn are easily mapped to the transcriptomic differential expression data. This, however, should not be seen as a quantitative measurement of fluxes, but rather as an indication of an activation or inactivation of certain parts of the network. First, we ran EBSeq (v3.12) ${ }^{112}$ on the gene count matrix generated in the previous steps. EBSeq returns the Posterior Probabilities of each gene being Differentially Expressed (PPDE) as well as the log2 fold changes. Then, we used the output of EBSeq as input to the Moomin method ${ }^{32}$ using default parameters. The Moomin method recovers topologically connected pathways predicted to be activated or inactivated based on the expression changes of corresponding GPRs included in the metabolic network. As there is usually not only one solution for a given differential expression dataset, a high number of solutions should be enumerated to construct a consensus solution. We enumerated 500 topological solutions for each of the datasets tested.

Isoform analysis. Using transcript quantification data from StringTie as input, we identified isoform switching events and their predicted functional consequences with the IsoformSwitchAnalyzeR R package (v1.11.3) ${ }^{113}$.

To calculate differential activity between samples, isoform usage is measured by the IF value, which quantifies the individual isoform expression level relative to the parent gene's expression level as previously presented in Eq. (1):

$$
\mathrm{IF}_{\text {isoform } 1}=\frac{\text { Isoform expression } 1}{\text { Gene expression (isoform expression } 1+\text { isoform } 2+\ldots \text { isoform } n \text { ) }}
$$

By proxy, the dIF between samples measures the effect size between conditions and is calculated as previously presented in Eq. (2):

$$
\mathrm{dIF}=\mathrm{IF}_{\text {condition } 2}-\mathrm{IF}_{\text {condition } 1}
$$

dIF was measured on a scale of 0 to 1 , with $0=$ no $(0 \%)$ change in usage between conditions and $1=$ complete $(100 \%)$ change in usage. The sum of dIF values for all isoforms associated with one gene is equal to 1 . We next filtered for isoforms that experienced $>30 \%$ switch in usage ( $\mathrm{dIF} \geq|0.3|$ ) and had an FDRcorrected $p$-value cutoff of $<0.05(q$-value $<0.05)$, which we define as "significant isoforms" for the remainder of the Methods.

Following filtering for these significant isoforms, we predicted their coding capabilities, protein structure stability, peptide signaling, and shifts in protein domain usage using The Coding-Potential Assessment Tool ${ }^{114}$, IUPred2 ${ }^{115}$, SignalP $\mathrm{P}^{116}$, and Pfam tools ${ }^{117}$, respectively. These external analyses were imported back into IsoformSwitchAnalyzeR and were used for downstream biological consequence and alternative splicing event enrichment analyses.

To plot individual isoform usage by differential gene expression, we combined the IsoformSwitchAnalyzeR dIF calculations and gene expression data from the aforementioned DESeq 2 results. The top 30 isoforms per dataset comparison were identified by ranking isoforms by gene switch $q$-value, i.e., the significance of the 
summation of all isoform switching events per gene between mock and infected conditions.

A biological consequence is defined as the biological property of a transcript (i.e., domain region, ORF, etc.). After calculating the number of significant isoforms experiencing a biological consequence or alternative splicing event, we performed enrichment analysis to determine if a consequence or splicing event occurred more frequently in a particular direction (i.e., gain vs. loss) across conditions. For example, a fraction score of 0.5 implies that out of all significant isoforms experiencing consequence $\mathrm{A}, 50 \%$ experience a gain in consequence $\mathrm{A}$ and $50 \%$ experience a loss in consequence $\mathrm{A}$, indicating no global preference in the direction the isoform population experiences consequence A. Statistical differences between consequence directions were calculated using a Fisher's exact test and $p$ values were FDR adjusted.

TE analysis. TE expression was quantified using the TEcount function from the TEtools software ${ }^{118}$. TEcount detects reads aligned against copies of each TE family annotated from the reference genome. DETEs in infected vs. mock conditions were detected using DEseq2 with a matrix of counts for genes and TE families as input. Functional enrichment of nearby genes (upstream $5 \mathrm{~kb}$ and downstream $1 \mathrm{~kb}$ of each TE copy within the human genome) was calculated with GREAT ${ }^{51}$ using options "genome background" and "basal + extension." Only the occurrences that were identified as statistically significant by region using a binomial test were selected.

\section{Identification of putative binding sites for human RBPs on the SARS-CoV-2} genome. The list of RBPs downloaded from ATtRACT was filtered to retain only human RBPs. PWMs for these RBPs were obtained from ATtRACT. The PWM is a representation of the experimentally determined sequence-binding preferences of the RBP. These PWMs were further filtered to retain PWMs obtained through competitive experiments and to drop PWMs with very high entropy. This left 205 experimentally determined PWMs for 102 human RBPs. The SARS-CoV-2 reference genome sequence was scanned with these 205 PWMs to detect sequence matches using the TFBSTools R package (v1.20.0). This scored sub-sequences on the genome based on their sequence match to the given PWMs. A minimum score threshold of $90 \%$ was used to identify putative RBP-binding sites.

Enrichment analysis for putative RBP-binding sites. The sequence of the SARS$\mathrm{CoV}-2$ genome was shuffled 1000 times. Each of the 1000 shuffled sequences was scanned for putative RBP-binding sites as described above. Next, the number of putative binding sites for each RBP was counted, and the mean and SD of the number of sites was calculated for each RBP across all 1000 shuffled sequences. The $z$-score for each RBP was then calculated as provided in Eq. (3):

$$
Z=\frac{\mathrm{S}_{\text {real }}-\overline{\mathrm{S}}_{\text {shuffled }}}{\sigma_{\text {shuffled }}}
$$

where $\mathrm{S}_{\text {real }}$ is the number of putative binding sites for the RBP on the real genome, $\overline{\mathrm{S}}_{\text {shuffled }}$ is the mean number of putative binding sites for the RBP across 1000 shuffled sequences, and $\sigma_{\text {shuffled }}$ is the SD of the number of putative binding sites for the RBP across 1000 shuffled sequences. The two-tailed $p$-value for each RBP was calculated from the $z$-score. A minimum FDR-adjusted $p$-value of 0.01 was taken as the cutoff for significant enrichment or depletion.

The same analysis was repeated taking only the sequence of the $5^{\prime}$-UTR, $3^{\prime}$ UTR, or intergenic regions of the SARS-CoV-2 reference genome, and was also repeated using the negative-sense genome sequence. Finally, this analysis was repeated with the reference genomes of SARS-CoV and RaTG13.

Conservation analysis for putative RBP-binding sites. The multiple sequence alignment of 181,003 SARS-CoV-2 genome sequences was downloaded from GISAID $^{119}$. For each putative RBP-binding site, we selected the corresponding columns of the multiple sequence alignment. We then counted the number of genomes in which the sequence was identical to that of the reference genome.

Viral genotype-phenotype association. All complete SARS-CoV-2 genomes having disease severity metadata in GISAID on 11 November 2020, together with the GenBank reference sequence, were aligned with MAFFT (v7.464) within a high-performance computing environment using 1 thread and the-nomemsave parameter ${ }^{120}$. Sequences responsible for introducing excessive gaps in this initial alignment were then manually identified and removed, leaving 8079 sequences that were then used to generate a new multiple sequence alignment.

The disease severity metadata for these sequences was then normalized into four categories: severe (862 samples), moderate (3873 samples), mild (2996 samples), and NA (310 samples). The complete correspondence between original patient status and these four categories can be found in Supplementary Data 17. These categories were based on whether the patient was treated in the intensive-care unit or died during acute infection, hospitalized or symptomatic, asymptomatic, not specified, or not available, respectively. The distribution of patient biological sex included males (4067 samples), females (3085 samples), unknown (798 samples), and not specified (129 samples). Patient age was converted into age ranges including the following: unspecified (38 samples), $<20$ (441 samples), 20-29 (1009 samples), 30-39 (1268 samples), 40-49 (1255 samples), 50-64 (1661), 65-74 (769), 75-84 (516 samples), >85 years old (283 samples), or not available (839 samples).

Next, the sequence data and associated metadata were used as input to the meta-CATS ${ }^{59}$ algorithm. Meta-CATS uses a $\chi^{2}$-statistical test to identify aligned positions containing significant differences in their base distribution between two or more metadata categories (e.g., severe vs. mild disease or male vs. female). The Benjamini-Hochberg multiple hypothesis correction was then applied to all positions ${ }^{121}$. Significant results were then evaluated against the annotated protein regions of the reference genome to determine their effect on amino acid sequence.

\section{Statistics and reproducibility}

RNA-seq datasets. Biological replicates for individual conditions are described as follows: within GSE147507 series 1, 2, 5, 7, 8, and 9 consisted of three biological replicates; series 3 and 4 consisted of two biological replicates. Within GSE150316, Cases 8 and 9 along with the negative control consisted of five biological replicates; Cases 1 and 4 consisted of four biological replicates; Cases 2 and 11 consisted of three biological replicates; and Case 3 consisted of two biological replicates. More details on the samples and replicates for each dataset are given in Supplementary Data 1.

DEGs and TEs. Differential expression of genes and TEs was separately determined based on the counts of features with DESeq2 (v1.26.0) ${ }^{103}$, which is based on a negative binomial regression model. The method normalized sequencing depth using a median-to-ratio method; then a Bayesian shrinkage approach was used to estimate both coefficients and dispersion parameters in the negative binomial. Then, a Wald's test was performed to identify DE genes or DETEs.

Enrichment analyses. The GO functional enrichment analysis was performed by retrieving all of the GO annotations for each DEG in each dataset. A hypergeometric statistical test was applied to all of the GO annotations for each DEG in each dataset, functions with an FDR-adjusted $p$-value $<0.05$ were considered significantly overrepresented.

Genes with FDR-adjusted $p$-values $<0.05$ based on DESeq2 were used as the gene list, whereas the superset of both significant and nonsignificant genes for each dataset was used as the background gene list. These lists of genes were then subjected to 5000 bootstrap replicates to generate a null distribution for each available pathway. Pathways that had a Bonferroni-adjusted $p$-value $<0.05$ were labeled as statistically significant and were reported in the results.

Differential usage of isoforms. IsoformSwitchAnalyzeR R package (v1.11.3) ${ }^{113}$ detected genome-wide enrichment by counting isoform switches and comparing the number of gain vs. losses. Enrichment tests were performed via base R's prop test and comparisons of enrichments were done with fisher.test. FDR-adjusted $p$ values ( $q$-values) inferior to 0.05 were considered significant.

Metabolic flux prediction. We ran EBSeq (v3.12) ${ }^{112}$ on the gene count matrix EBSeq returned the posterior probabilities of each gene being differentially expressed (PPDE) and the $\log 2$ fold changes. Moomin ${ }^{32}$ then used the results of PPDE along with $\log 2$ fold changes to predict topological solutions within the metabolic network.

TE functional enrichment analysis. Based on DETEs, we used GREAT ${ }^{51}$ to analyze the functional significance of cis-regulatory regions. The method performs a binomial test on the total portion of the genome associated with any given ontology vs. the fraction of the input genomic regions which fall into those areas.

RBP analysis. Enrichment or depletion of putative RBP-binding sites in a sequence was calculated by shuffling the sequence 1000 times and scanning each shuffled sequence for putative RBP-binding sites. A $z$-score for each RBP was calculated as provided in Eq. (3):

$$
Z=\frac{\mathrm{S}_{\text {real }}-\overline{\mathrm{S}}_{\text {shuffled }}}{\sigma_{\text {shuffled }}}
$$

where $S_{\text {real }}$ is the number of putative binding sites for the RBP on the real genome, $\bar{S}_{\text {shuffled }}$ is the mean number of putative binding sites for the RBP across 1000 shuffled sequences, and $\sigma_{\text {shuffled }}$ is the SD of the number of putative binding sites for the RBP across 1000 shuffled sequences. The two-tailed $p$-value for each RBP was calculated from the $z$-score. A minimum FDR-adjusted $p$-value of 0.01 was taken as the cutoff for significant enrichment or depletion.

Viral genotype-phenotype association. Over 8000 SARS-CoV-2 genomes that had associated clinical metadata such as disease severity, age, or biological sex were included in the analysis. The sequences were divided into categories based on the available clinical metadata before being subjected to a $\chi^{2}$-statistical test. Results that met an FDR-adjusted $p$-value $<0.05$ were labeled as statistically significant and were manually reviewed to identify aligned positions that had the potential for 
statistical skew due to not surpassing the minimal number of bases in a given category (at least five viral strains having the same base in each category).

Reporting summary. Further information on research design is available in the Nature Research Reporting Summary linked to this article.

\section{Data availability}

RNA-seq datasets with accessions GSE147507 and GSE150316 were obtained from the NCBI Gene Expression Omnibus (GEO) database (https://www.ncbi.nlm.nih.gov/geo). The reference genome sequences of SARS-CoV-2, RaTG13, and SARS-CoV were downloaded from Genbank under the accessions NC_045512, MN996532.1, and NC_004718.3, respectively. A list of known RNA-binding proteins (RBPs) and their Position Weight Matrices (PWMs) were downloaded from ATtRACT (https://attract. cnic.es/download). Normalized gene expression values in human lung tissue were obtained from the GTEx database, version 8 (https://gtexportal.org/home/datasets). Single-cell RNA-seq data for human lung cells were obtained from the NCBI GEO database under accession GSE122960. Finally, all SARS-CoV-2 complete genomes collected from humans, along with associated metadata, were downloaded from the GISAID database (https://www.gisaid.org/) on 11 November $2020^{119}$. Supplementary data have been deposited on Zenodo at https://doi.org/10.5281/zenodo.4644596 ${ }^{122}$. Any other data are available from the corresponding authors on reasonable request.

\section{Code availability}

Code for the analyses described in this work is available at https:/github.com/ vaguiarpulido/covid19-research ${ }^{123}$, under the MIT open-source license. The following versions of software were used in this study: STAR (v2.3.7a), Samtools (v1.10), StringTie (v2.1.1), DESeq2 (v1.26.0), GOstats package (v2.54.0), IsoformSwitchAnalyzeR R package (v1.11.3), Moomin (v1.0), EBSeq (v3.12), TETools (v1.0.0), sra-tools (v2.10.8), FastQC (v0.11.9), MultiQC (v1.9), TFBSTools R package (v1.20.0), and MAFFT (v7.464).

Received: 20 August 2020; Accepted: 5 April 2021;

Published online: 17 May 2021

\section{References}

1. Huang, C. et al. Clinical features of patients infected with 2019 novel coronavirus in Wuhan, China. Lancet 395, 497-506 (2020).

2. Andersen, K. G., Rambaut, A., Lipkin, W. I., Holmes, E. C. \& Garry, R. F. The proximal origin of SARS-CoV-2. Nat. Med. 26, 450-452 (2020).

3. WHO Coronavirus (COVID-19) Dashboard. https://covid19.who.int.

4. Tsang, K. W. et al. A cluster of cases of severe acute respiratory syndrome in Hong Kong. N. Engl. J. Med. 348, 1977-1985 (2003).

5. Zaki, A. M., van Boheemen, S., Bestebroer, T. M., Osterhaus, A. D. M. E. \& Fouchier, R. A. M. Isolation of a novel coronavirus from a man with pneumonia in Saudi Arabia. N. Engl. J. Med. 367, 1814-1820 (2012).

6. Zhou, P. et al. A pneumonia outbreak associated with a new coronavirus of probable bat origin. Nature 579, 270-273 (2020).

7. Lam, T. T.-Y. et al. Identifying SARS-CoV-2-related coronaviruses in Malayan pangolins. Nature 583, 282-285 (2020)

8. Ingraham, N. E. et al. Immunomodulation in COVID-19. Lancet Respir. Med. 8, 544-546 (2020).

9. Beigel, J. H. et al. Remdesivir for the treatment of Covid-19 - final report. $N$. Engl. J. Med. 383, 1813-1826 (2020).

10. Standl, F., Jöckel, K.-H., Brune, B., Schmidt, B. \& Stang, A. Comparing SARSCoV-2 with SARS-CoV and influenza pandemics. Lancet Infect. Dis. https:// doi.org/10.1016/S1473-3099(20)30648-4 (2020).

11. Wang, Y., Wang, Y., Chen, Y. \& Qin, Q. Unique epidemiological and clinical features of the emerging 2019 novel coronavirus pneumonia (COVID-19) implicate special control measures. J. Med. Virol. 92, 568-576 (2020).

12. Giamarellos-Bourboulis, E. J. et al. Complex immune dysregulation in COVID-19 patients with severe respiratory failure. Cell Host Microbe 27, 992-1000.e3 (2020).

13. Yan, R. et al. Structural basis for the recognition of SARS-CoV-2 by full-length human ACE2. Science 367, 1444-1448 (2020).

14. Fung, T. S. \& Liu, D. X. Human coronavirus: host-pathogen interaction. Annu. Rev. Microbiol. 73, 529-557 (2019).

15. Li, Z. \& Nagy, P. D. Diverse roles of host RNA binding proteins in RNA virus replication. RNA Biol 8, 305-315 (2011).

16. Macchietto, M. G., Langlois, R. A. \& Shen, S. S. Virus-induced transposable element expression up-regulation in human and mouse host cells. Life Sci. Alliance 3, e201900536 (2020).

17. Chuong, E. B., Elde, N. C. \& Feschotte, C. Regulatory activities of transposable elements: from conflicts to benefits. Nat. Rev. Genet. 18, 71-86 (2017).
18. Gordon, D. E. et al. A SARS-CoV-2 protein interaction map reveals targets for drug repurposing. Nature 583, 459-468 (2020).

19. Daniloski, Z. et al. Identification of required host factors for SARS-CoV-2 infection in human cells. Cell https://doi.org/10.1016/j.cell.2020.10.030 (2020)

20. Wen, W. et al. Immune cell profiling of COVID-19 patients in the recovery stage by single-cell sequencing. Cell Discov. 6, 31 (2020).

21. Liao, M. et al. Single-cell landscape of bronchoalveolar immune cells in patients with COVID-19. Nat. Med. 26, 842-844 (2020).

22. Blanco-Melo, D. et al. Imbalanced host response to SARS-CoV-2 drives development of COVID-19. Cell 181, 1036-1045.e9 (2020).

23. Chasman, D. et al. Integrating transcriptomic and proteomic data using predictive regulatory network models of host response to pathogens. PLoS Comput. Biol. 12, e1005013 (2016).

24. The Gene Ontology Consortium. The Gene Ontology Resource: 20 years and still GOing strong. Nucleic Acids Res. 47, D330-D338 (2019).

25. Zhang, F. et al. Adaptive immune responses to SARS-CoV-2 infection in severe versus mild individuals. Signal Transduct. Target Ther. 5, 156 (2020).

26. Zhang, J.-Y. et al. Single-cell landscape of immunological responses in patients with COVID-19. Nat. Immunol. 21, 1107-1118 (2020).

27. Wilk, A. J. et al. A single-cell atlas of the peripheral immune response in patients with severe COVID-19. Nat. Med. 26, 1070-1076 (2020).

28. Thiele, I. et al. A community-driven global reconstruction of human metabolism. Nat. Biotechnol. 31, 419-425 (2013).

29. Metallo, C. M. \& Heiden, M. G. V. Understanding metabolic regulation and its influence on cell physiology. Mol. Cell 49, 388 (2013).

30. Watson, E., Yilmaz, L. S. \& Walhout, A. J. Understanding metabolic regulation at a systems level: metabolite sensing, mathematical predictions, and model organisms. Annu. Rev. Genet. 49, 553-75 (2015).

31. Patel, M. S. \& Harris, R. A. Metabolic Regulation. in Encyclopedia of Cell Biology: Volume 1 (eds. Bradshaw, R. A. \& Stahl, P. D.) 288-297 (Academic Press, 2016)

32. Pusa, T. et al. MOOMIN - Mathematical explOration of Omics data on a MetabolIc Network. Bioinformatics 36, 514-523 (2020).

33. Wang, R. et al. Genetic Screens Identify Host Factors for SARS-CoV-2 and Common Cold Coronaviruses. Cell 184, 106-119.e14, https://doi.org/10.1016/ j.cell.2020.12.004 (2021).

34. Leppek, K., Das, R. \& Barna, M. Functional 5' UTR mRNA structures in eukaryotic translation regulation and how to find them. Nat. Rev. Mol. Cell Biol. 19, 158-174 (2018).

35. Mignone, F., Gissi, C., Liuni, S. \& Pesole, G. Untranslated regions of mRNAs. Genome Biol. 3, REVIEWS0004 (2002).

36. Goodarzi, H. et al. Systematic discovery of structural elements governing stability of mammalian messenger RNAs. Nature 485, 264-268 (2012).

37. de Koning, A. P. J., Gu, W., Castoe, T. A., Batzer, M. A. \& Pollock, D. D. Repetitive elements may comprise over two-thirds of the human genome. PLoS Genet. 7, e1002384 (2011).

38. Cordaux, R. \& Batzer, M. A. The impact of retrotransposons on human genome evolution. Nat. Rev. Genet. 10, 691-703 (2009).

39. Mills, R. E., Bennett, E. A., Iskow, R. C. \& Devine, S. E. Which transposable elements are active in the human genome? Trends Genet. 23, 183-191 (2007).

40. Sundaram, V. \& Wysocka, J. Transposable elements as a potent source of diverse -regulatory sequences in mammalian genomes. Philos. Trans. R. Soc. Lond. B Biol. Sci 375, 20190347 (2020).

41. Friedli, M. \& Trono, D. The developmental control of transposable element and the evolution of higher species. Annu. Rev. Cell Dev. Biol. 31, 429-451 (2015).

42. Babaian, A. \& Mager, D. L. Endogenous retroviral promoter exaptation in human cancer. Mob. DNA 7, 24 (2016).

43. Kojima, K. K. Human transposable elements in Repbase: genomic footprints from fish to humans. Mob. DNA 9, 2 (2018).

44. Pace, J. K. 2nd \& Feschotte, C. The evolutionary history of human DNA transposons: evidence for intense activity in the primate lineage. Genome Res. 17, 422-432 (2007).

45. Smit, A. F. Interspersed repeats and other mementos of transposable elements in mammalian genomes. Curr. Opin. Genet. Dev. 9, 657-663 (1999).

46. Chuong, E. B., Elde, N. C. \& Feschotte, C. Regulatory evolution of innate immunity through co-option of endogenous retroviruses. Science 351, 1083-1087 (2016).

47. Schmid, C. D. \& Bucher, P. MER41 repeat sequences contain inducible STAT1 binding sites. PLoS ONE 5, e11425 (2010).

48. Deniz, Ö. et al. Endogenous retroviruses are a source of enhancers with oncogenic potential in acute myeloid leukaemia. Nat. Commun. 11, 3506 (2020).

49. Ito, J. et al. Systematic identification and characterization of regulatory elements derived from human endogenous retroviruses. PLoS Genet. 13 e1006883 (2017).

50. van de Lagemaat, L. N., Landry, J.-R., Mager, D. L. \& Medstrand, P. Transposable elements in mammals promote regulatory variation and 
diversification of genes with specialized functions. Trends Genet. 19, 530-536 (2003).

51. McLean, C. Y. et al. GREAT improves functional interpretation of cisregulatory regions. Nat. Biotechnol. 28, 495-501 (2010).

52. Giudice, G., Sánchez-Cabo, F., Torroja, C. \& Lara-Pezzi, E. ATtRACT-a database of RNA-binding proteins and associated motifs. Database 2016, baw035 (2016)

53. Kim, D. et al. The Architecture of SARS-CoV-2 Transcriptome. Cell 181, 914-921.e10 (2020).

54. GTEx Consortium. Human genomics. The Genotype-Tissue Expression (GTEx) pilot analysis: multitissue gene regulation in humans. Science 348, 648-660 (2015).

55. Reyfman, P. A. et al. Single-cell transcriptomic analysis of human lung provides insights into the pathobiology of pulmonary fibrosis. Am. J. Respir. Crit. Care Med. 199, 1517-1536 (2019).

56. Mukherjee, N. et al. Integrative regulatory mapping indicates that the RNAbinding protein $\mathrm{HuR}$ couples pre-mRNA processing and mRNA stability. Mol. Cell 43, 327-339 (2011)

57. Ladd, A. N., Charlet, N. \& Cooper, T. A. The CELF family of RNA binding proteins is implicated in cell-specific and developmentally regulated alternative splicing. Mol. Cell. Biol. 21, 1285-1296 (2001).

58. Jurica, M. S., Licklider, L. J., Gygi, S. R., Grigorieff, N. \& Moore, M. J. Purification and characterization of native spliceosomes suitable for threedimensional structural analysis. RNA 8, 426-439 (2002).

59. Pickett, B. E. et al. Metadata-driven comparative analysis tool for sequences (meta-CATS): an automated process for identifying significant sequence variations that correlate with virus attributes. Virology 447, 45-51 (2013)

60. Mehta, H. M., Malandra, M. \& Corey, S. J. G-CSF and GM-CSF in neutropenia. J. Immunol. 195, 1341-1349 (2015).

61. Wu, D. \& Yang, X. O. TH17 responses in cytokine storm of COVID-19: An emerging target of JAK2 inhibitor Fedratinib. J. Microbiol. Immunol. Infect. 53, 368-370 (2020).

62. Mehta, P. et al. COVID-19: consider cytokine storm syndromes and immunosuppression. Lancet 395, 1033-1034 (2020).

63. Zhou, Y. \& Zhu, Y. Important role of the IL-32 inflammatory network in the host response against viral infection. Viruses 7, 3116-3129 (2015).

64. Vitting-Seerup, K. \& Sandelin, A. The landscape of isoform switches in human cancers. Mol. Cancer Res. 15, 1206-1220 (2017).

65. Gandal, M. J. et al. Transcriptome-wide isoform-level dysregulation in ASD, schizophrenia, and bipolar disorder. Science 362, eaat8127 (2018).

66. Tazi, J., Bakkour, N. \& Stamm, S. Alternative splicing and disease. Biochim. Biophys. Acta. 1792, 14-26 (2009).

67. Saulle, I. et al. A new ERAP2/Iso3 isoform expression is triggered by different microbial stimuli in human cells. Could it play a role in the modulation of SARS-CoV-2 infection? Cells 9, 1951 (2020).

68. Zhang, Z.-L., Hou, Y.-L., Li, D.-T. \& Li, F.-Z. Laboratory findings of COVID19: a systematic review and meta-analysis. Scand. J. Clin. Lab. Invest. 80, 441-447 (2020).

69. Aziz, M., Fatima, R. \& Assaly, R. Elevated interleukin-6 and severe COVID19: a meta-analysis. J. Med. Virol. 92, 2283-2285 (2020).

70. Chen, G. et al. Clinical and immunological features of severe and moderate coronavirus disease 2019. J. Clin. Invest. 130, 2620-2629 (2020).

71. Huang, L. et al. Sepsis-associated severe interleukin-6 storm in critical coronavirus disease 2019. Cell. Mol. Immunol. 17, 1092-1094 (2020).

72. Grifoni, E. et al. Interleukin-6 as prognosticator in patients with COVID-19. J. Infect. 81, 452-482 (2020).

73. McElvaney, O. J. et al. A linear prognostic score based on the ratio of interleukin-6 to interleukin-10 predicts outcomes in COVID-19. EBioMedicine 61, 103026 (2020).

74. Hassan, N. \& Choy, E. in Oxford Textbook of Rheumatoid Arthritis 389-398 (Oxford Univ. Press, 2020)

75. Horváth, S. \& Mirnics, K. Immune system disturbances in schizophrenia. Biol. Psychiatry 75, 316-323 (2014).

76. Nitto, T. \& Onodera, K. Linkage between coenzyme a metabolism and inflammation: roles of pantetheinase. J. Pharmacol. Sci. 123, 1-8 (2013).

77. Guo, W. et al. SNX8 modulates the innate immune response to RNA viruses by regulating the aggregation of VISA. Cell. Mol. Immunol. https://doi.org/ 10.1038/s41423-019-0285-2 (2019).

78. Hinz, M. et al. Signal responsiveness of IкB kinases is determined by Cdc37assisted transient interaction with Hsp90. J. Biol. Chem. 282, 32311-32319 (2007).

79. Li, J.-Y. et al. The ORF6, ORF8 and nucleocapsid proteins of SARS-CoV-2 inhibit type I interferon signaling pathway. Virus Res. 286, 198074 (2020).

80. Trizzino, M., Kapusta, A. \& Brown, C. D. Transposable elements generate regulatory novelty in a tissue-specific fashion. BMC Genomics 19, 468 (2018).

81. Soliman, S., Faris, M. E., Ratemi, Z. \& Halwani, R. Switching host metabolism as an approach to dampen SARS-CoV-2 infection. Ann. Nutr. Metab. 76, 297-303 (2020).
82. Abu-Farha, M. et al. The role of lipid metabolism in COVID-19 virus infection and as a drug target. Int. J. Mol. Sci. 21, 3544 (2020).

83. Song, J.-W. et al. Omics-driven systems interrogation of metabolic dysregulation in COVID-19 pathogenesis. Cell Metab. 32, 188-202.e5 (2020).

84. Thomas, T. et al. COVID-19 infection alters kynurenine and fatty acid metabolism, correlating with IL-6 levels and renal status. JCI Insight 5, e140327 (2020).

85. Flynn, R. A. et al. Discovery and functional interrogation of SARS-CoV-2 RNAhost protein interactions. Cell https://doi.org/10.1016/j.cell.2021.03.012 (2021).

86. Schmidt, N. et al. The SARS-CoV-2 RNA-protein interactome in infected human cells. Nat Microbiol 6, 339-353 (2021).

87. Smith, R. W. P. \& Gray, N. K. Poly(A)-binding protein (PABP): a common viral target. Biochem. J 426, 1-12 (2010).

88. Perez, C., McKinney, C., Chulunbaatar, U. \& Mohr, I. Translational control of the abundance of cytoplasmic poly(A) binding protein in human cytomegalovirus-infected cells. J. Virol. 85, 156-164 (2011).

89. Ahlquist, P., Noueiry, A. O., Lee, W.-M., Kushner, D. B. \& Dye, B. T. Host factors in positive-strand RNA virus genome replication. J. Virol 77, 8181-8186 (2003)

90. Polacek, C., Friebe, P. \& Harris, E. Poly(A)-binding protein binds to the nonpolyadenylated $3^{\prime}$ untranslated region of dengue virus and modulates translation efficiency. J. Gen. Virol 90, 687-692 (2009).

91. Smith, R. W. P. et al. Viral and cellular mRNA-specific activators harness PABP and eIF4G to promote translation initiation downstream of cap binding. Proc. Natl Acad. Sci. USA 114, 6310-6315 (2017).

92. Li, H. P., Zhang, X., Duncan, R., Comai, L. \& Lai, M. M. Heterogeneous nuclear ribonucleoprotein A1 binds to the transcription-regulatory region of mouse hepatitis virus RNA. Proc. Natl Acad. Sci. USA 94, 9544-9549 (1997)

93. Huang, P. \& Lai, M. M. Heterogeneous nuclear ribonucleoprotein al binds to the $3^{\prime}$-untranslated region and mediates potential $5^{\prime}-3^{\prime}$-end cross talks of mouse hepatitis virus RNA. J. Virol. 75, 5009-5017 (2001).

94. Shi, S. T., Huang, P., Li, H. P. \& Lai, M. M. Heterogeneous nuclear ribonucleoprotein A1 regulates RNA synthesis of a cytoplasmic virus. EMBO J. 19, 4701-4711 (2000).

95. Pachetti, M. et al. Emerging SARS-CoV-2 mutation hot spots include a novel RNA-dependent-RNA polymerase variant. J. Transl. Med. 18, 179 (2020).

96. Kannan, S. R. et al. Infectivity of SARS-CoV-2: there is something more than D614G? J. Neuroimmune Pharmacol. 15, 574-577 (2020).

97. Hillen, H. S. et al. Structure of replicating SARS-CoV-2 polymerase. Nature 584, 154-156 (2020).

98. Wang, R., Hozumi, Y., Zheng, Y.-H., Yin, C. \& Wei, G.-W. Host immune response driving SARS-CoV-2 evolution. Viruses 12, 1095 (2020).

99. Ewels, P., Magnusson, M., Lundin, S. \& Käller, M. MultiQC: summarize analysis results for multiple tools and samples in a single report. Bioinformatics 32, 3047-3048 (2016).

100. Dobin, A. et al. STAR: ultrafast universal RNA-seq aligner. Bioinformatics 29, 15-21 (2013)

101. Li, H. et al. The Sequence Alignment/Map format and SAMtools. Bioinformatics 25, 2078-2079 (2009).

102. Pertea, M. et al. StringTie enables improved reconstruction of a transcriptome from RNA-seq reads. Nat. Biotechnol. 33, 290-295 (2015).

103. Love, M. I., Huber, W. \& Anders, S. Moderated estimation of fold change and dispersion for RNA-seq data with DESeq2. Genome Biol. 15, 550 (2014).

104. Anders, S. \& Huber, W. Differential expression analysis for sequence count data. Genome Biol. 11, R106 (2010).

105. Ashburner, M. et al. Gene ontology: tool for the unification of biology. The Gene Ontology Consortium. Nat. Genet. 25, 25-29 (2000).

106. Falcon, S. \& Gentleman, R. Using GOstats to test gene lists for GO term association. Bioinformatics 23, 257-258 (2007).

107. Lesack, K. \& Naugler, C. An open-source software program for performing Bonferroni and related corrections for multiple comparisons. J. Pathol. Inform. 2, 52 (2011).

108. Supek, F., Bošnjak, M., Škunca, N. \& Šmuc, T. REVIGO summarizes and visualizes long lists of gene ontology terms. PLoS ONE 6, e21800 (2011).

109. Tarca, A. L. et al. A novel signaling pathway impact analysis. Bioinformatics 25, 75-82 (2009).

110. Sales, G., Calura, E., Cavalieri, D. \& Romualdi, C. graphite - a Bioconductor package to convert pathway topology to gene network. BMC Bioinformatics 13, 20 (2012).

111. Huang, D. W., Sherman, B. T. \& Lempicki, R. A. Systematic and integrative analysis of large gene lists using DAVID bioinformatics resources. Nat. Protoc. 4, 44-57 (2009).

112. Leng, N. et al. EBSeq: an empirical Bayes hierarchical model for inference in RNA-seq experiments. Bioinformatics 29, 1035-1043 (2013).

113. Vitting-Seerup, K. \& Sandelin, A. IsoformSwitchAnalyzeR: analysis of changes in genome-wide patterns of alternative splicing and its functional consequences. Bioinformatics 35, 4469-4471 (2019). 
114. Wang, L. et al. CPAT: Coding-Potential Assessment Tool using an alignmentfree logistic regression model. Nucleic Acids Res. 41, e74 (2013).

115. Dosztányi, Z., Csizmok, V., Tompa, P. \& Simon, I. IUPred: web server for the prediction of intrinsically unstructured regions of proteins based on estimated energy content. Bioinformatics 21, 3433-3434 (2005).

116. Almagro Armenteros, J. J. et al. SignalP 5.0 improves signal peptide predictions using deep neural networks. Nat. Biotechnol. 37, 420-423 (2019).

117. El-Gebali, S. et al. The Pfam protein families database in 2019. Nucleic Acids Res. 47, D427-D432 (2019).

118. Lerat, E., Fablet, M., Modolo, L., Lopez-Maestre, H. \& Vieira, C. TEtools facilitates big data expression analysis of transposable elements and reveals an antagonism between their activity and that of piRNA genes. Nucleic Acids Res. 45, e17 (2017).

119. Shu, Y. \& McCauley, J. GISAID: Global initiative on sharing all influenza data - from vision to reality. Euro. Surveill. 22, 30494 (2017).

120. Nakamura, T., Yamada, K. D., Tomii, K. \& Katoh, K. Parallelization of MAFFT for large-scale multiple sequence alignments. Bioinformatics 34, 2490-2492 (2018).

121. Benjamini, Y. \& Hochberg, Y. Controlling the false discovery rate: a practical and powerful approach to multiple testing. J. R. Stat. Soc. Ser. B Methodol. 57, 289-300 (1995).

122. Ferrarini, M. G. et al. Supplementary materials for 'Genome-wide bioinformatic analyses predict key host and viral factors in SARS-CoV-2 pathogenesis', https://doi.org/10.5281/zenodo.4644596 (2021).

123. Ferrarini, M. G. et al. vaguiarpulido/covid19-research: covid19-research, https://doi.org/10.5281/zenodo.4576990 (2021).

\section{Acknowledgements}

We thank the Virtual BioHackathon on COVID-19 that took place during April 2020 (https://github.com/virtual-biohackathons/covid-19-bh20) for fostering an environment that triggered this collaboration. We also thank Slack for providing us with free access to the professional version of the platform. We gratefully acknowledge the GISAID database, together with the various originating and submitting laboratories that were responsible for generating the viral genome sequence data, and the associated metadata that were included in this study. R.R. thanks Dixie Mager for insightful discussions on TE analyses. The authors received no specific funding to support this work.

\section{Author contributions}

V.A.-P., A.G., and M.G.F. performed differential expression analyses and GO enrichment analyses. J.S., E.B., and B.E.P. performed pathway enrichment analysis. M.G.F. and T.P. performed metabolic flux analysis. T.F. and V.A.-P. performed isoform differential usage analysis. R.R., M.G.F., D.S.O., and V.A.-P. performed transposable element analyses. A.L. performed analyses of RNA-binding proteins with advice from A.J.G. B.E.P. performed analyses on viral sequence variants. I.M.G. advised with discussions on immunology. All authors wrote and approved the manuscript.

\section{Competing interests}

The authors declare no competing interests.

\section{Additional information}

Supplementary information The online version contains supplementary material available at https://doi.org/10.1038/s42003-021-02095-0.

Correspondence and requests for materials should be addressed to B.E.P. or V.A-P.

Reprints and permission information is available at http://www.nature.com/reprints

Publisher's note Springer Nature remains neutral with regard to jurisdictional claims in published maps and institutional affiliations.

(c) (i) Open Access This article is licensed under a Creative Commons Attribution 4.0 International License, which permits use, sharing, adaptation, distribution and reproduction in any medium or format, as long as you give appropriate credit to the original author(s) and the source, provide a link to the Creative Commons license, and indicate if changes were made. The images or other third party material in this article are included in the article's Creative Commons license, unless indicated otherwise in a credit line to the material. If material is not included in the article's Creative Commons license and your intended use is not permitted by statutory regulation or exceeds the permitted use, you will need to obtain permission directly from the copyright holder. To view a copy of this license, visit http://creativecommons.org/ licenses/by/4.0/.

(c) The Author(s) 2021 